\title{
Itô calculus and quantum white noise calculus
}

\author{
Luigi Accardi ${ }^{1}$ and Andreas Boukas ${ }^{2}$ \\ 1 Centro Vito Volterra, Università di Roma Tor Vergata via Columbia, 2- 00133 \\ Roma, Italy. accardi@Volterra.mat.uniroma2.it, \\ http://volterra.mat.uniroma2.it \\ 2 Department of Mathematics and Natural Sciences, American College of Greece, \\ Aghia Paraskevi, Athens 15342, Greece, andreasboukas@acgmail.gr.
}

Summary. Itô calculus has been generalized in white noise analysis and in quantum stochastic calculus. Quantum white noise calculus is a third generalization, unifying the two above mentioned ones and bringing some unexpected insight into some old problems studied in different fields, such as the renormalization problem in physics and the representation theory of Lie algebras. The present paper is an attempt to explain the motivations of these extensions with emphasis on open challenges.

The last section includes a result obtained after the Abel Symposium. Namely that, after introducing a new renormalization technique, the RHPWN Lie algebra includes (in fact we will prove elsewhere that this inclusion is an identification) a second quantized version of the extended Virasoro algebra, i.e. the Virasoro-Zamolodchikov *-Lie algebra $w_{\infty}$, which has been widely studied in string theory and in conformal field theory. ${ }^{3}$

\section{Introduction}

The year 2005 marks Kiyosi Itô's 90th birthday and, with it, the 63th birthday of stochastic calculus. The present Abel symposium, devoted to the celebration of these events, offers to all mathematicians an important occasion to meditate on this important development in their discipline whose influence is going to follow the times of history, even in a period when the pace of scientific development has reached a level in which most papers have a life time of less than one year.

The applications of Itô's work have been so many, ranging from physics to biology, from logistics and operation research to engineering, from meteorology to mathematical finance, ..., that an exhaustive list is impossible.

\footnotetext{
${ }^{3}$ Talk given by L.A. at the Abel symposium: Stochastic analysis and applications: A symposium in honor of Kiyosi Itô (on the occasion of his 90th birthday) Oslo, Friday 29 July-Thursday 4 August, 2005 Organized by: the Norwegian Mathematical Society and the Center of Mathematics for Applications (CMA), Oslo
} 
From the mathematical point of view it is someteimes underestimated the fact that Itô calculus, with its radical innovation of the two basic operations of calculus - differentiation and integration - has been one of the few real conceptual breakthroughs in the development of classical analysis after Newton.

Itô laid down the foundations of stochastic calculus in his 1942 thesis [Itô42a], [Itô42b] and the first systematic exposition of these ideas in English language appeared almost ten years later in [Itô51] and preceeded of about 15 years the now classical monograph [ItôMcKn65]. This gave rise to an impetuous development which has seen as protagonists several of the participants to the present conference and which will be reviewed by them.

My talk will take the move from one of the basic achievements of this development, completed in the late 1960's, and which led to the mathematical substantiation of a limpid and intuitive picture of the structure of a classical stochastic process indexed by the real line (interpreted as time) and with values in $\mathbb{R}^{d}$ (interpreted as a generalized phase space).

The sample space of a generic process of this type is identified to a space of $\mathbb{R}^{d}$-valued functions, interpreted as trajectories of a dynamical system, and each trajectory is canonically decomposed into a sum of two parts: a regular (bounded variation) part, corresponding to the drift in the stochastic equation and a pure fluctuation term, corresponding to the martingale part in the stochastic equation. The former part is handled with classical, Newtonian, calculus; the latter with Itô calculus. The picture is completed by the Kunita-Watanabe martingale representation theorem [KunWat67], which characterizes the generic martingales as stochastic integrals with respect to some stationary, independent increment process and by the Lévy-Itô decomposition of a stationary, independent increment process $\left(Z_{t}\right)$ :

$$
Z_{t}=m t+\sigma B_{t}+X_{t}
$$

where $m$ is a constant, $B_{t}$ is a Brownian motion and $X_{t}$ is a compound Poisson process, i.e. an integral

$$
X_{T}=\int_{0}^{T} d t \int P_{u, t} d \beta(u)
$$

of independent Poisson processes $P_{u, t}$ with intensity of jumps equal to $u$, with respect to a measure $d \beta(u)$, called the Levy measure and with support in $\mathbb{R} \backslash\{0\}$.

The early generalizations of Itô calculus had gone in the direction of extending it to more general state spaces thus passing from $\mathbb{R}^{d}$ to manifolds or to infinite dimensions or both. Another, less developed extension was from vector valued to operator valued classical stochastic processes [Skor84]. However these extensions did not change the basic conceptual framework of the theory.

The situation changed in the past 30 years when three qualitative innovations appeared. This drastically enlarged not only the conceptual status of 
Itô calculus, and more generally of stochastic analysis, but also its technical apparatus. The traditional bridges between probability, classical analysis and combinatorics became an intricate network including practically every field of mathematics, from operator theory to graph theory, from Hopf algebras to group representations, $\ldots$

The traditional applications to the classical world (physics, information, communications, engineering, finance,...) have now been expanded to the corresponding sectors in the quantum world thus bringing a remedy to the historical paradox according to which the mathematical discipline, dealing with the laws of chance, was not powerful enough to include into its framework the most advanced physical theory, quantum mechanics, in which chance enters in a much more intrinsic way than in any other physical theory.

These innovations begun with two, initially quite separated and independent, lines of research: white noise analysis, (1975) and quantum stochastic calculus (1982) and found their unification, starting from 1993, in quantum white noise calculus.

The rate of progression of these events, as well as the merging of different generalizations into a single, unified picture, has been so swift that, even for those who actively participated in the construction of these developments, it is quite hard to follow all the new ideas and to embrace the whole landscape in a single eyesight.

It is precisely on this broad picture that the present paper will be focused. Not only details, but also several important achievements, will be omitted from the exposition, in the attempt to convey an idea of some of the exciting new perspectives of quantum stochastic analysis.

The first attempts to go beyond the Itô calculus framework and to include processes which, although much more singular, were frequently used in the physics and engineering literature, was Hida white noise theory, first proposed in his Carleton lectures of 1975 [Hida75], [Hida92].

The second conceptual generalization of Itô calculus took place in 1982 when Hudson and Parthasarathy developed their quantum stochastic calculus [HuPa82a], [HuPa84c]. In it for the first time, the noises themselves (i.e. the martingales driving the stochastic differential equations) were no longer classical additive independent increment processes but quantum independent increment processes. This was the first quantum generalization of Itô calculus and opened the way to all subsequent ones. The culmination of the theory is the determination of the structure of those stochastic equations which admit a unitary solution. The reason why this result has fundamental implications both for quantum mechanics and for classical probability, will be explained starting from section (7).

The Hudson-Parthasarathy theory inspired, directly or indirectly, most of the developments of quantum probability for the decade after its appearance. Its importance can be compared to the original Itô paper and the multiplicity of investigations it motivated was surveyed in [Partha92]. 
But the story does not end here: a third conceptual generalization, motivated by the stochastic limit of quantum theory, was developed between 1993 and 1995 and can be described as the unification of the white noise and the quantum stochastic approach: the non triviality of this unification will be clear starting from section (12) of the present exposition. In particular this third step threw a new and unexpected light on the microscopic structure of quantum, hence in particular classical, stochastic equations as a consequence of:

(i) the discovery of the Hamiltonian structure of the (classical and quantum) stochastic differential equations

(ii) the discovery of the translation code between white noise and stochastic differential equations. This required the development of the theory of distributions on the standard simplex [AcLuVo99] which is the mathematical counterpart of the time consecutive principle of the stochastic limit of quantum theory.

However the main point of the new development was not so much the deeper understanding of the structure of classical and quantum stochastic calculus, but the possibilities it opened of further extensions, which cannot be obtained with the traditional tools of stochastic analysis. In fact the white noise extension of the Itô table opened the way to the nonlinear generalizations of Itô calculus to which is devoted the second part of the present report.

The beautiful landscape emerging from the simplest of these extensions, i.e. the one dealing with the second power of white noise, and the subsequent, totally unexpected unification of the five Meixner classes as classical subprocesses (algebraically: Cartan $*$-sub algebras) respectively of the first and second order white noise, rose strong hopes that this hierarchy could be extended from the second powers of white noise to its higher powers. This would lead to a new, interesting class of infinitely divisible processes (for a short while there was even the hope to obtain a new parametrization of all these processes).

This hope however collided with the wall of the no go theorems described in the last part of the present paper. Although negative results, these theorems are very interesting because they have revealed an hitherto unknown phenomenon relating stochastic analysis to two different fields, each of which has been the object of a huge literature outside probability theory namely:

(i) the representation theory of infinite dimensional Lie algebras

(ii) renormalization theory.

These two theories are at the core of contemporary theoretical physics and the fact that some developments, motivated by quantum white noise analysis, could bring new insight and new results in such a fundamental issue, which resisted decades of efforts from the best minds of theoretical physics, is an indication that this direction is deep and worth being pursued. For this reason while the first part of the present paper consists in an exposition of already established results, in the second part emphasis has been laid on the formulation of the problems facing the construction of a satisfactory theory 
of the higher powers of white noise. This has led to the introduction of some new notions, such as Fock representation of a Lie algebra, which are going to play an essential role in the development of the theory.

All these developments show that Itô calculus shares, with the richest and deepest mathematical theories, the germs of its radical innovation. Historical experience shows that these innovations often occur in directions which are quite unexpected for the experts of the field and this sometimes generates a feeling of extraneousness.

An instructive example is given by the theory of elliptic functions, originated from a deep intuition of Abel and initially developed within a purely analytical context, but now stably settled in a purely algebraic and geometrical framework.

The story we are going to tell shows that Itô calculus gives another important example in this direction.

\section{Plan of the present paper}

The goal of the present section is twofold: (i) to give a more analytical outline of the content of the present paper; (ii) to catch this occasion to say a few words about the motivations and the inner logic underlying the developments described here as well as about their connections with other sectors of quantum probability which could not be dealt with for reasons of space.

Section (3) defines the notion of quantum (Boson Fock) white noise and illustrates, in this basic particular case, one of the main ideas of quantum probability, i.e. the idea that algebra implies statistics. Let me just mention here that also the converse statement, i.e. that statistics implies algebra (e.g. commutation or anti commutation relations), is true and it lies at a deeper level. The first result in this direction was proved by von Waldenfels in the Bose and Fermi case [voWaGi78], [voWa78] and about 20 years later, with the introduction of the notion of interacting Fock space [AcLuVo97b], this principle became a quite universal principle of probability theory and opened the way to the program of a full algebraic classification of probability measures. This is a quite interesting direction, and is also deeply related to the main topic of the present paper, stochastic and white noise calculus, but we will not discuss this connection and we refer the interested reader to [AcBo98], [AcKuSt02], [AcKuSt05a].

Section (4) describes another important new idea of quantum probability, i.e. the notion of quantum decomposition of a classical random variable (or stochastic process). This idea is illustrated in the important particular case of classical white noise and extended, in Section (6), to the Poisson noise.

The two above mentioned decompositions are at the root of HudsonParthasarathy's quantum extension of classical Itô calculus, briefly outlined in Section (6). 
Section (7) briefly describes the classical Schrödinger and Heisenberg equations as a preparation to their stochastic and white noise versions.

The algebraic form of a classical stochastic process is described in section (8). This leads to a reformulation, explained in section (10), of classical stochastic differential equations, that makes quite transparent their equivalence to stochastic versions of the classical Schrödinger or of Heisenberg equations.

In sections (9), (10) it is briefly outlined how this reformulation is nothing but a stochastic analogue of Koopman's algebraization of the theory of classical, deterministic dynamical systems.

Combining the content of section (8) with the quantum decomposition of classical white and Poisson noise, described in sections (4), (6), one arrives, in section (11), to the full quantum versions of the stochastic Schrödinger and Heisenberg equations, which are the main object of study of the HudsonParthasarathy theory.

These equations are not of Hamiltonian type and they were developed by Hudson and Parthasarathy on the basis of a purely mathematical analogy with the classical Itô calculus. Hence their connection with the Hamiltonian equations of quantum physics was obscure and the early applications of these equations to physical problems, proposed by Barchielli [Barc88], Belavkin [Bela86a], Gardiner and Collet [GaCo85], ..., were built on a purely phenomenological basis. This led to some misgivings among physicists on the meaning of these models and their relations to the fundamental laws of quantum mechanics.

On the other hand, combining the main results of Hudson and Parthasarathy (construction of unitary Markovian cocycles) with the quantum Feynman-Kac formula of $[\mathrm{Ac} 78 \mathrm{~b}]$ we see that, by quantum conditioning of a stochastic Heisenberg evolution $X_{0} \mapsto U_{t} X_{0} U_{t}^{*}$ on the time zero algebra, one obtains quantum Markov semigroup $\left(P^{t}\right)$ :

$$
E_{0]}\left(U_{t} X_{0} U_{t}^{*}\right)=P^{t}\left(X_{0}\right)
$$

just as the analogue classical conditioning leads to a classical Markov semigroup. It was also known, since the early results of Pauli and van Hove, that quantum Markov semigroups $\left(P^{t}\right)$ (and the associated master equations, which are the quantum analogue of the Chapman-Kolmogorov equations) can arise as appropriate time-scaling limits of reduced Heisenberg evolutions. The time-scaling being the same one used in classical stochastic homogenization (i.e. $t \rightarrow t / \lambda^{2}$ ), and known in the physical literature as van Hove or $1 / \lambda^{2}$ scaling, and the limit being taken for $\lambda \rightarrow 0$. Since (in this particular context) the physical operation of reducing an Heisenberg evolution to a subsystem, used in these papers, is mathematically equivalent to conditioning on the time zero algebra, the above statement can be rewritten as:

$$
\lim _{\lambda \rightarrow 0} E_{0]}\left(U_{t / \lambda^{2}}^{(\lambda)} X_{0} U_{t / \lambda^{2}}^{(\lambda) *}\right)=P^{t}\left(X_{0}\right)
$$


Comparing (1) with (2) it was therefore natural to conjecture that also the unconditioned limits,

$$
\begin{gathered}
\lim _{\lambda \rightarrow 0} U_{t / \lambda^{2}}^{(\lambda)} X_{0} U_{t / \lambda^{2}}^{(\lambda) *}=U_{t} X_{0} U_{t}^{*} \\
\lim _{\lambda \rightarrow 0} U_{t / \lambda^{2}}^{(\lambda)}=U_{t}
\end{gathered}
$$

of the original Schrödinger and Heisenberg evolutions should exist, for some (at those times unspecified) topology, and satisfy some quantum stochastic Schrödinger and Heisenberg equations of Hudson-Parthasarathy type.

This conjecture was formulated by Frigerio and Gorini immediately after the development of quantum stochastic calculus [FrGo82a] and was proved a few years later by Accardi, Frigerio and Lu [AcFrLu87].

This result marked the beginning of the stochastic limit of quantum theory. It proved that quantum stochastic differential equations arise as physically meaningful scaling and limiting procedures from the fundamental laws of quantum mechanics, expressed in terms of Hamiltonian equations. This produced, among other things, a microscopic interpretation not only of the coefficients of the stochastic equations, but also of the fine structure of the driving martingales (quantum noises).

Several years later Accardi, Lu and Volovich [AcLuVo93] realized that in fact stochastic differential equations (both classical and quantum) are themselves Hamiltonian equations but not of usual type: they are white noise Hamiltonian equations. The identification of these two classes of equations required the development of new mathematical techniques such as the notion of causal normal order and the strictly related time consecutive principle and theory of distributions on the standard simplex (cf. [AcLuVo02] for a discussion of these notions).

The inclusion: classical and quantum $S D E \subseteq W N$ Hamiltonian equations is a consequence of this development and is described in Sections (12), (13). These few pages condensate a series of developments which took place in several years and in several papers. The interested reader is referred to [AcLuVo99] (the first attempt to systematize the impetuous development of the previous years) and to the more recent expositions [Ayed05] (thesis of Wided Ayed) and the papers [AcAyOu03], [AcAyOu05a], [AcAyOu05b]. The last of this papers deals with another one of the several interesting developments born from the stochastic limit of quantum theory which, for lack of space, are not discussed in the present paper, namely the module generalization of white noise calculus and the qualitatively new structure of the quantum noises emerging from it (the reader, interested in the first and main physical example of this new structure, is referred to [AcLuVo97b]).

Even more condensed is the description, in sections (14), (15), (16), (17), of the renormalized square of WN. This is because the survey paper [AcBou04c] is specifically devoted to this subject and the interested reader can find there the necessary information. 
On the contrary, since most of the material in sections from (18) to (22) has not yet been published, we tried to give all the necessary definitions even if proofs had to be omitted for reasons of space.

The general problem, concerning the renormalized higher powers of $\mathrm{WN}$, is formulated in section (21) with the related no-go theorems. As explained in sections (22), this problem is also related with an old open problem of classical probability, i.e. the infinite divisibility of the odd powers of a standard Gaussian random variable.

Further investigations are needed to understand the effective impact of these no-go theorems. Do they really close the hope of a general theory of higher powers of white noise? Our feeling is that the answer to this question is no! This hope is supported by the following considerations. The no-go theorems heavily depend on:

(i) the choice of a renormalization procedure;

(ii) the fact that we restrict our attention to a very special representation, i.e. the Fock one.

A way out of this conundrum has to be looked for in the relaxation of one of these assumption, i.e. one has to look for either new renormalization procedures or different representations. Both ways are now under investigation and raise challenging but fascinating mathematical problems.

The last section of the present paper refers to a development that took place after the end of the Abel Symposium and which shows that the idea to look for different types of renormalization procedures turned out particularly fruitful and brought to the fore a connection between the renormalized higher powers of white noise and the Virasoro algebra which promises to be as rich of developments as the connection between the renormalized square of white noise and the Meixner classes.

\section{Fock scalar White Noise (WN)}

Definition 1. The standard d-dimensional Fock scalar White Noise (WN) is defined by a quadruple

$$
\left\{\mathcal{H}, b_{t}, b_{t}^{+}, \Phi\right\} ; \quad t \in \mathbb{R}^{d}
$$

where $\mathcal{H}$ is a Hilbert space, $\Phi \in \mathcal{H}$ a unit vector called the (Fock) vacuum, and $b_{t}, b_{t}^{+}$are operator valued distributions (for an explanation of this notion see the comment at the end of the present section and the discussion in [AcLuVo02], section (2.1)) with the following properties.

The vectors of the form

$$
b_{t_{n}}^{+} \ldots b_{t_{1}}^{+} \Phi
$$

called the number vectors are well defined in the distribution sense and total in $\mathcal{H}$.

$b_{t}$ is the adjoint of $b_{t}^{+}$on the linear span of the number vectors 


$$
\left(b_{t}^{+}\right)^{+}=b_{t}
$$

Weakly on the same domain and in the distribution sense:

$$
\left[b_{s}, b_{t}^{+}\right]:=b_{s} b_{t}^{+}-b_{t}^{+} b_{s}=\delta(t-s)
$$

where, here and in the following, the symbol [ ·, · ] will denote the commutator:

$$
[A, B]:=A B-B A
$$

Finally $b_{t}$ and $\Phi$ are related by the Fock property (always meant in the distribution sense):

$$
b_{t} \Phi=0
$$

The unit vector $\Phi$ determines the expectation value

$$
\langle\Phi, X \Phi\rangle=:\langle X\rangle
$$

which is well defined for any operator $X$ acting on $\mathcal{H}$ and with $\Phi$ in its domain.

Remark. In the Fock case algebra implies statistics in the sense that the algebraic rules (7), (6), (8) uniquely determine the restriction of the expectation value $(9)$ on the polynomial algebra generated by $b_{t}$ and $b_{t}^{+}$. This is because, with the notation

$$
X^{\varepsilon}=\left\{\begin{array}{l}
X, \varepsilon=-1 \\
X^{*}, \varepsilon=+1
\end{array}\right.
$$

the Fock prescription (8) implies that the expectation value

$$
\left\langle b_{t_{n}}^{\varepsilon_{n}} \ldots b_{t_{1}}^{\varepsilon_{1}}\right\rangle
$$

of any monomial in $b_{t}$ and $b_{t}^{+}$is zero whenever either $n$ is odd or $b_{t_{1}}^{\varepsilon_{1}}=b_{t_{1}}$ or $b_{t_{n}}^{\varepsilon_{n}}=b_{t_{n}}^{+}$. If neither of these conditions is satisfied, then there is a $k \in$ $\{2, \ldots, n\}$ such that the expectation value (11) is equal to

$$
\left\langle b_{t_{n}}^{\varepsilon_{n}} \ldots b_{t_{1}}^{\varepsilon_{1}}\right\rangle=\left\langle b_{t_{n}}^{\varepsilon_{n}} \ldots b_{t_{k+1}}^{\varepsilon_{k+1}}\left[b_{t_{k}}, b_{t_{k-1}}^{+} \ldots b_{t_{1}}^{+}\right]\right\rangle
$$

Using the derivation property of the commutator $\left[b_{t_{k}}, \cdot\right]$ (i.e. $\left.(24)\right)$ one then reduces the expectation value (12) to a linear combination of expectation values of monomials of order less or equal than $n-2$. Iterating one sees that only the scalar term can give a nonzero contribution.

Remark. The practical rule to deal with operator valued distributions is the following: products of the form (11) are meant in the sense that, after multiplication by $\varphi\left(t_{n}\right) \cdot \ldots \cdot \varphi\left(t_{1}\right)$, where $\varphi_{1}, \ldots, \varphi_{n}$ are elements of an appropriate test function space (typically one chooses the space of smooth functions decreasing at infinity faster than any polynomial), and integration with respect to all variables $d t_{1} \cdot \ldots \cdot d t_{n}$ (each of which runs over $\mathbb{R}^{d}$ ) one obtains a product of well defined operators whose products contain the vector $\Phi$ in their domains. Here and in the following we will not repeat each time when an identity has to be meant in the distribution sense. 


\section{Classical real valued white noise}

Lemma Let $b_{t}, b_{t}^{+}$be a Fock scalar white noise. Then

$$
w_{t}:=b_{t}+b_{t}^{+}
$$

is a classical real random variable valued distribution satisfying:

$$
\begin{gathered}
w_{t}=w_{t}^{+} \\
{\left[w_{s}, w_{t}\right]=0 \quad ; \quad \forall s, t} \\
\left\langle w_{t}\right\rangle=0 \\
\left\langle w_{s} w_{t}\right\rangle=\delta(t-s) \\
\left\langle w_{t_{2 n}} \ldots w_{t_{1}}\right\rangle=\sum_{\left\{l_{\alpha}, r_{\alpha}\right\} \in p . p .\{1, \ldots, 2 n\}} \prod_{\alpha=1}^{n}\left\langle w_{t_{l_{\alpha}}} w_{t_{r_{\alpha}}}\right\rangle
\end{gathered}
$$

moreover all odd moments vanish.

Remark. The self-adjointness condition (14) and the commutativity condition (15) mean that $\left(w_{t}\right)$ is (isomorphic to) a classical real valued process. Conditions (16) and (17) mean respectively that $\left(w_{t}\right)$ is mean zero and $\delta$ correlated. Finally (18), which follows from (8) and from the same arguments used to deduce the explicit form of $(11)$, shows that the classical process $\left(w_{t}\right)$ is Gaussian.

Definition 2. The process $\left(w_{t}\right)$ satisfying (14),..., (17) (one can prove its uniqueness up to stochastic equivalence) is called the standard $d$-dimensional classical real valued White Noise (WN). The identity (13) is called the quantum decomposition of the classical d-dimensional white noise.

Remark. Notice that, for the classical process $\left(w_{t}\right)$, it is not true that algebra implies statistics: this becomes true only using the quantum decomposition (13) combined with the Fock prescription (8).

Remark. In the case $d=1$, integrating the classical WN one obtains the classical Brownian motion with zero initial condition:

$$
W_{t}=B_{t}+B_{t}^{+}=\int_{0}^{t} d s\left(b_{s}^{+}+b_{s}\right)
$$

Notice that (19) gives the $q$-decomposition of the classical BM just as (13) gives the $q$-decomposition of the classical WN.

From now on we will only consider the case $d=1$. 


\section{Classical subprocesses associated to the first order white noise}

An important generalization of the quantum decomposition (13) of the classical white noise is the identity:

$$
p_{t}(\lambda)=b_{t}+b_{t}^{+}+\lambda b_{t}^{+} b_{t} ; \quad \lambda \geq 0
$$

which can be shown to define (in the sense of vacuum distribution) a 1parameter family of classical real valued distribution processes (i.e. $p_{t}(\lambda)=$ $p_{t}(\lambda)^{+}$and $\left.\left[p_{s}(\lambda), p_{t}(\lambda)\right]=0\right)$. In fact this classical process can be identified, up to a time rescaling, to the compensated scalar valued standard classical Poisson noise with intensity $1 / \lambda$ and the identity (20) gives a $q$-decomposition of this process.

Integrating (20), in analogy with (19), one obtains the standard compensated Poisson processes. Notice that the critical value

$$
\lambda=0
$$

corresponds to the classical $\mathrm{WN}$ while any other value

$$
\lambda \neq 0
$$

gives a Poisson noise. As a preparation to the discussion of Section (17) notice that $\lambda=0$ is the only critical point, i.e. a point where the vacuum distribution changes and that these two classes of stochastic processes exactly coincide with the first two Meixner classes.

\section{The Hudson-Parthasarathy quantum stochastic calculus}

In the previous sections we have seen that, integrating the densities

$$
\begin{gathered}
w_{t}=b_{t}+b_{t}^{+} \\
p(\lambda)_{t}=b_{t}+b_{t}^{+}+\lambda b_{t}^{+} b_{t}
\end{gathered}
$$

one obtains the stochastic differentials (random measures) as WN integrals

$$
\begin{gathered}
d W_{t}=\int_{t}^{t+d t} w_{s} d s=\int_{t}^{t+d t}\left(b_{s}+b_{s}^{+}\right) d s=: d B_{t}^{+}+d B_{t} \\
d P_{t}(\lambda)=\int_{t}^{t+d t} p_{s}(\lambda) d s=\int_{t}^{t+d t}\left(b_{s}+b_{s}^{+}+\lambda b_{s}^{+} b_{s}\right) d s=d B_{t}^{+}+d B_{t}+\lambda d N_{t}
\end{gathered}
$$


Starting from these one defines the classical stochastic integrals with the usual constructions.

$$
\int_{0}^{t} F_{s} d W_{s} \quad ; \quad \int_{0}^{t} F_{s} d P_{s}(\lambda)
$$

The passage to $q$-stochastic integrals consists in separating the stochastic integrals corresponding to the different pieces. In other words, the quantum decomposition (20) suggests to introduce separately the stochastic integrals

$$
\int_{0}^{t} F_{s} d B_{s} ; \quad \int_{0}^{t} F_{s} d B_{s}^{+} ; \quad \int_{0}^{t} F_{s} d N_{s}
$$

This important development was due to Hudson and Parthasarathy and we refer to the monograph [Partha92] for an exposition of the whole theory.

\section{Schrödinger and Heisenberg equations}

A Schrödinger equation (also called an operator Hamiltonian equation) is an equation of the form:

$$
\partial_{t} U_{t}=-i H_{t} U_{t} \quad ; \quad U_{0}=1 \quad ; \quad t \in \mathbb{R}
$$

where the 1 -parameter family of symmetric operators on a Hilbert space $\mathcal{H}$

$$
H_{t}=H_{t}^{*}
$$

is called the Hamiltonian. In the pyhsics literature one often requires the positivity of $H_{t}$. We do not follow this convenction in order to give a unified treatment of the usual Schrödinger equation and of its so-called interaction representation form. This approach is essential to underline the analogy with the white noise Hamiltonian equations, to be discussed in section (12).

When $H_{t}$ is a self-adjoint operator independent of $t$, the solution of equation (21) exists and is a 1-parameter group of unitary operators:

$$
U_{t} \in U n(\mathcal{H}) ; U_{s} U_{t}=U_{s+t} ; U_{0}=1 ; U_{t}^{*}=U_{t}^{-1}=U_{-t} ; s, t \in \mathbb{R}
$$

Conversely every 1-parameter group of unitary operators is the solution of equation (21) for some self-adjoint operator $H_{t}=H$ independent of $t$.

An Heisenberg equation, associated to equation (21), is

$$
\partial_{t} X_{t}=\delta_{t}\left(X_{t}\right) \quad ; \quad X_{0}=X \in \mathcal{B}(\mathcal{H})
$$

where $\delta_{t}$ has the form

$$
\delta_{t}\left(X_{t}\right):=-i\left[H_{t}, X_{t}\right] \quad ; \quad X_{0}=X \in \mathcal{B}(\mathcal{H})
$$

One can prove that $\delta_{t}$ is a $*$-derivation, i.e. a linear operator on an appropriate subspace of the algebra $\mathcal{B}(\mathcal{H})$ of all the bounded operators on $\mathcal{H}$, also called the algebra of observables, satisfying (on this subspace): 


$$
\begin{gathered}
\delta_{t}(a b)=\delta_{t}(a) b+a \delta_{t}(b) \\
\delta_{t}^{*}(a):=\delta_{t}\left(a^{*}\right)^{*}=\delta_{t}(a)
\end{gathered}
$$

Not all $*$-derivations $\delta_{t}$ on subspaces (or sub algebras) of $\mathcal{B}(\mathcal{H}$ ) have the form (23). If this happens, then the $*$-derivation, $\delta_{t}$, and sometimes also the Heisenberg equation, is called inner and its solution has the form

$$
X_{t}=U_{t} X_{t} U_{t}^{*}
$$

where $U_{t}$ is the solution of the corresponding Schrödinger equation (21). Conversely, every solution $U_{t}$ of the Schrödinger equation (21) defines, through (25), a solution of the Heisenberg equation (22) with $\delta_{t}$ given by (23).

Thus every Schrödinger equation is canonically associated to an Heisenberg equation. The converse is in general false, i.e. there are Heisenberg equations with no associated Schrödinger equation (equivalently: not always a derivation is inner). The simplest physically relevant examples of this situation are given by the quantum generalization of the so called interacting particle systems [AcKo00b] which have been widely studied in classical probability.

\section{Algebraic form of a classical stochastic process}

Let $\left(X_{t}\right)$ be a real valued stochastic process. Define

$$
j_{t}(f):=f\left(X_{t}\right)
$$

In the spirit of quantum probability, we realize $f$ as a multiplication operator on $L^{2}(\mathbb{R})$ and $f\left(X_{t}\right)$ as a multiplication operator on

$$
L^{2}\left(\mathbb{R} \times \Omega, \mathcal{B}_{\mathbb{R}} \times \mathcal{F}, d x \otimes P\right) \equiv L^{2}(\mathbb{R}) \otimes L^{2}(\Omega, \mathcal{F}, P)
$$

where $(\Omega, \mathcal{F}, P)$ is the probability space of the process $\left(X_{t}\right)$ and $\mathcal{B}_{\mathbb{R}}$ denotes the Borel $\sigma$-algebra on $\mathbb{R}$. Sometimes we use the notation:

$$
M_{f} \varphi(x):=f(x) \varphi(x) \quad ; \quad \varphi \in L^{2}(\mathbb{R})
$$

The same notation will be used if $x \in \mathbb{R}$ is replaced by $(x, \omega) \in \mathbb{R} \times \Omega$.

Thus $f\left(X_{t}\right)$ is realized as multiplication operator on $L^{2}(\mathbb{R}) \otimes L^{2}(\Omega, \mathcal{F}, P)$. With these notations, for each $t \geq 0, j_{t}$ is a $*$-homomorphism

$$
j_{t}: \mathcal{C}^{2}(\mathbb{R}) \subseteq \mathcal{B}\left(L^{2}(\mathbb{R})\right) \rightarrow \mathcal{B}\left(L^{2}(\mathbb{R}) \otimes L^{2}(\Omega, \mathcal{F}, P)\right)
$$




\section{Koopman's argument and quantum extensions of classical deterministic dynamical systems}

The following considerations, due to Koopman, constitute the basis of the algebraic approach to dynamical systems which reduces the study of such systems to the study of 1 -parameter groups of unitary operators or of $*-$ automorphisms of appropriate commutative $*$-algebras or, at infinitesimal level, to the study of appropriate Schrödinger or Heisenberg equations.

To every ordinary differential equation in $\mathbb{R}^{d}$

$$
d x_{t}=b\left(x_{t}\right) d t \quad ; \quad x(0)=x_{0} \in \mathbb{R}^{d}
$$

such that the initial value problem admits a unique solution for every initial data $x_{0}$ and for every $t \geq 0$ : one associates the 1 -parameter family of maps

$$
T_{t}: \mathbb{R}^{d} \rightarrow \mathbb{R}^{d}
$$

characterized by the property that the image of $x_{0}$ under $T_{t}$ is the value of the solution at time $t$ :

$$
x_{t}\left(x_{0}\right)=: T_{t} x_{0} \quad ; \quad T_{0}=i d
$$

Uniqueness then implies the semigroup property:

$$
T_{t} T_{s}=T_{t+s}
$$

If the above properties hold not only for every $t \geq 0$, but for every $t \in \mathbb{R}$, then the system is called reversible. In this case each $T_{t}$ is invertible and

$$
T_{t}^{-1}=T_{-t}
$$

Typical examples of these systems are the classical Hamiltonian systems. They have the additional property that the maps $T_{t}$ preserve the Lebesgue measure (Liouville's theorem).

Abstracting the above notion to an arbitrary measure space leads to the notion of (deterministic) dynamical system:

Definition 3. Let $(S, \mu)$ be a measure space. A classical, reversible, deterministic dynamical system is a pair:

$$
\left\{(S, \mu) ;\left(T_{t}\right) t \in \mathbb{R}\right\}
$$

where $T_{t}: S \rightarrow S(t \in \mathbb{R})$ is a 1-parameter group of invertible bi-measurable maps of $(S, \mu)$ admitting $\mu$ as a quasi-invariant measure:

$$
\mu \circ T_{t} \sim \mu
$$


The quasi-invariance of $(S, \mu)$ is equivalent to the existence of a $\mu$-almost everywhere invertible Radon-Nikodym derivative:

$$
\begin{gathered}
\frac{d\left(\mu \circ T_{t}\right)}{d \mu}=: p_{\mu, t} \in L^{1}(S, \mu) \\
p_{\mu, t}>0 ; \mu-\text { a.e.; } \quad \int_{S} p_{\mu, t}(s) d \mu(s)=1
\end{gathered}
$$

Therefore for any $t \in \mathbb{R}$ the linear map

$$
\varphi \mapsto U_{t} \varphi(s):=p_{\mu, t}(s)^{-1 / 2} \varphi\left(T_{t}^{-1} s\right)
$$

is well defined for any measurable function $\varphi$. Moreover

$$
\begin{gathered}
\int_{S}\left(p_{\mu, t}(s)\right)^{-1 / 2} \bar{f}\left(T_{t}^{-1}(s)\right)\left(p_{\mu, t}(s)\right)^{-1 / 2} g\left(T_{t}^{-1}(s)\right) d \mu(s)= \\
=\int_{S} \bar{f}\left(T_{t}^{-1} s\right) g\left(T_{t}^{-1} s\right) p_{\mu, t}(s)^{-1} d \mu(s) \\
\quad=\int_{S} \bar{f}\left(T_{t}^{-1} s\right) g\left(T_{t}^{-1} s\right) p_{\mu, t}\left(T_{t} T_{t}^{-1} s\right)^{-1} d \mu\left(T_{t} T_{t}^{-1} s\right)
\end{gathered}
$$

and since

$$
d \mu\left(T_{t} T_{t}^{-1} s\right)=\frac{d \mu T_{t}}{d \mu}\left(T^{-1} s\right) d \mu\left(T^{-1} s\right)
$$

the change of variables

$$
T_{t}^{-1} s=s^{\prime}
$$

gives

$$
\left\langle U_{t} f, U_{t} g\right\rangle=\int_{S} \bar{f}\left(s^{\prime}\right) g\left(s^{\prime}\right) d \mu\left(s^{\prime}\right)=\langle f, g\rangle
$$

i.e. the map

$$
U_{t}: f \in L^{2}(S, \mu) \rightarrow p_{\mu, t}^{-1 / 2} f \circ T_{t}^{-1} \in L^{2}(S, \mu)
$$

defines a unitary operator in $L^{2}(S, \mu)$. Similarly one proves that the family $\left(U_{t}\right)$ is a 1-parameter unitary group:

$$
U_{t} U_{s}=U_{t+s} \quad ; \quad U_{t}^{*}=U_{-t} \quad ; \quad U_{0}=i d
$$

By Stone's theorem there exists a self-adjoint operator $H$, on $L^{2}(S, \mu)$, which is the infinitesimal generator of this 1-parameter group, i.e.

$$
U_{t}=e^{-i t H}
$$

and this is equivalent to the Schrödinger equation

$$
\partial_{t} U_{t}=-i H U_{t} \quad ; \quad U_{0}=1
$$


The unitarity implies that:

$$
U_{t}^{*} \varphi=U_{t}^{-1} \varphi=p_{\mu, t}(s)^{1 / 2} \varphi \circ T_{t}
$$

therefore, recalling that $M_{f}$ denotes the multiplication operator by $f\left(M_{f} \varphi=\right.$ $f \varphi)$, one has

$$
\begin{aligned}
& \left(U_{t} M_{f} U_{t}^{*} \varphi\right)(s)=\left(p_{\mu, t}\right)(s)^{-1 / 2}\left(M_{f} U_{t}^{*} \varphi\right)\left(T_{t}^{-1} s\right) \\
& \quad=p_{\mu, t}(s)^{-1 / 2} f\left(T_{t}^{-1} s\right) p_{\mu, t}(s)^{1 / 2} \varphi(s)=f\left(T_{t}^{-1} s\right) \varphi(s)
\end{aligned}
$$

In conclusion: the Heisenberg evolution on $\mathcal{B}\left(L^{2}(S, \mu)\right)$ :

$$
x \mapsto U_{t} x U_{t}^{*}=: j_{t}(x)
$$

canonically associated to the family $\left(U_{t}\right)$, satisfies the identity:

$$
M_{f \circ T_{t}^{-1}}=U_{t} M_{f} U_{t}^{*}=: j_{t}\left(M_{f}\right)
$$

This implies that the Abelian algebra $L^{\infty}(S, \mu)$ (considered as a sub-algebra of on $\left.\mathcal{B}\left(L^{2}(S, \mu)\right)\right)$ is left invariant by each $j_{t}$ :

$$
j_{t}\left(L^{\infty}(S, \mu)\right)=U_{t} L^{\infty}(S, \mu) U_{t}^{*} \subseteq L^{\infty}(S, \mu)
$$

Replacing the multiplication operator $M_{f}$ by an arbitrary bounded operator $x$, acting on $L^{2}(S, \mu)$ one obtains a quantum extension of a classical deterministic system. Abstraction from this situation suggests the following definition:

Definition 4. A deterministic, reversible, dynamical system is a pair:

$$
\left\{\mathcal{A} ;\left(j_{t}\right) t \in \mathbb{R}\right\}
$$

where $\mathcal{A}$ is a $*$-algebra and $\left(j_{t}\right)$ a 1-parameter group of automorphisms of $\mathcal{A}$. If $\mathcal{A}$ is a $C^{*}\left(W^{*}\right)$ algebra, then one speaks of a $C^{*}\left(W^{*}\right)$ dynamical system. If $\mathcal{A}$ is Abelian the system is called classical; otherwise it is called quantum. Finally, if $\mathcal{A}$ is non Abelian but it contains an Abelian sub-algebra $\mathcal{A}_{c l}$ left invariant by $j_{t}\left(j_{t}\left(\mathcal{A}_{c l}\right) \subseteq \mathcal{A}\right)$ then it is called a quantum extension of a classical system.

\section{Stochastic extension of Koopman's approach: emergence of Schrödinger and Heisenberg equations in classical stochastic analysis}

In the present section we will replace, in the above Koopman's argument, the deterministic trajectory $\left(x_{t}\left(x_{0}\right)\right)$ by a stochastic process $\left(X_{t}\right)$ and show how the general algebraization procedure described in section (8), when applied to 
the simple and important example of a classical diffusion flow $\left(X_{t}\right)$, naturally leads to a classical stochastic generalization of the Heisenberg equation.

Let $\left(X_{t}\right)$ denote the real valued solution of the classical stochastic differential equation

$$
d X_{t}=l d t+a d W_{t} \quad ; X(0)=X_{0}
$$

driven by classical Brownian motion $\left(W_{t}\right)$ and with adapted coefficients $l, a$ which guarantee the existence and uniqueness of a strong solution for all initial data $X_{0}$ in $L^{2}(\mathbb{R})$ and for all times. The initial value $X_{0}$ is a random variable independent of $\left(W_{t}\right)$. By Itô's formula equation (26) is equivalent to

$$
d f\left(X_{t}\right)=\left(l \partial_{x} f+\frac{1}{2} a^{2} \partial_{x}^{2} f\right) d t+a \partial_{x} f d W_{t}
$$

where $f: \mathbb{R} \rightarrow \mathbb{R}$ varies in a space of sufficiently smooth functions.

Since $X_{t}$ depends also on the initial condition $x \in \mathbb{R}, f\left(X_{t}\right)$ is realized as multiplication operator on

$$
L^{2}(\mathbb{R}) \otimes L^{2}(\Omega, \mathcal{F}, P)
$$

where $(\Omega, \mathcal{F}, P)$ is the probability space of the increment process of the Brownian motion. In the following we shall simply write $f$ or $\left.f\left(X_{t}\right)\right)$ to mean the multiplication operator by $f\left(\right.$ or $\left.f\left(X_{t}\right)\right)$. When confusion can arise we shall write $M_{f}$ or $M_{f\left(X_{t}\right)}$. With these notations one has:

$$
\left[\partial_{x}, f\right]=\left[\partial_{x}, M_{f}\right]=\partial_{x} \cdot f-f \cdot \partial_{x}=\partial_{x} f=M_{\partial_{x} f}
$$

Therefore

$$
\left[\partial_{x},\left[\partial_{x}, f\right]\right]=\left[\partial_{x},\left[\partial_{x}, M_{f}\right]\right]=M_{\partial_{x}^{2} f}=\Delta f=M_{\Delta f}
$$

Introducing the momentum operator on $L^{2}(\mathbb{R})$ :

$$
p:=\frac{1}{i} \partial_{x}
$$

defined on those functions in $L^{2}(\mathbb{R})$ with a derivative also in $L^{2}(\mathbb{R})$, we can write

$$
\partial_{x} f=i[p, f] \quad ; \quad \partial_{x}^{2} f=-[p,[p, f]]
$$

More generally, interpreting both $f$ and $l$ as multiplication operators and using the fact that $f$ commutes with $l$, one finds:

$$
\begin{gathered}
l f^{\prime}=l \partial_{x} f=l i[p, f]=\frac{i}{2} l[p, f]+\frac{i}{2}[p, f] l= \\
=\frac{i}{2} l p f-\frac{i}{2} l f p+\frac{i}{2} p f l-\frac{i}{2} f p l=i\left[\frac{1}{2} l p+\frac{1}{2} p l, f\right]=: i[p(l), f]
\end{gathered}
$$


and therefore:

$$
a^{2} \partial_{x}^{2} f=a \partial_{x} a \partial_{x} f-a\left(\partial_{x} a\right) \partial_{x} f=-\left[p(a),[p(a), f]-i\left[p\left(a \partial_{x} a\right), f\right]\right.
$$

Thus, with $p(a)$ and $p(l)$ defined by (28) equation (27) can be written in the form

$$
\begin{gathered}
d f\left(X_{t}\right)=i\left[p(a), f\left(X_{t}\right)\right] d W_{t}+ \\
+\left(i\left[p(l), f\left(X_{t}\right)\right]-\frac{1}{2}\left[p(a),\left[p(a), f\left(X_{t}\right)\right]\right]-i\left[p\left(\frac{1}{4} \partial_{x} a^{2}\right), f\left(X_{t}\right)\right]\right) d t
\end{gathered}
$$

In conclusion, denoting

$$
l+\frac{1}{2} \partial_{x} a^{2}=: l_{1}
$$

we write equation $(27)$ in the form

$$
d f\left(X_{t}\right)=i\left[p(a), f\left(X_{t}\right)\right] d W_{t}-\frac{1}{2}\left[p(a),\left[p(a), f\left(X_{t}\right)\right]\right] d t+i\left[p\left(l_{1}\right), f\left(X_{t}\right)\right] d t
$$

and, recalling the notation $f\left(X_{t}\right)=j_{t}(f)$, this is equivalent to

$$
d j_{t}(f)=i\left[p(a), j_{t}(f)\right] d W_{t}-\frac{1}{2}\left[p(a),\left[p(a), j_{t}(f)\right]\right] d t+i\left[p\left(l_{1}\right), j_{t}(f)\right] d t
$$

Since any operator acting on $L^{2}(\mathbb{R})$ can be identified to the operator $T \otimes 1$, acting on $L^{2}(\mathbb{R}) \otimes L^{2}(\Omega, \mathcal{F}, P)$, also the multiplication operators by functions $f$ in $\mathcal{C}^{2}(\mathbb{R})$, can be realized as pre-closed operators acting on $L^{2}(\mathbb{R}) \otimes L^{2}(\Omega, \mathcal{F}, P)$. With this identification, the homomorphisms $j_{t}$ can be considered as maps

$$
\begin{aligned}
j_{t}: \mathcal{C}^{2}(\mathbb{R}) \otimes 1 \subseteq \mathcal{B}\left(L^{2}(\mathbb{R})\right) \otimes 1 \subseteq \mathcal{B}\left(L^{2}(\mathbb{R})\right) \otimes & \mathcal{B}\left(L^{2}(\Omega, \mathcal{F}, P)\right) \\
& \equiv \mathcal{B}\left(L^{2}(\mathbb{R}) \otimes\left(L^{2}(\Omega, \mathcal{F}, P)\right)\right.
\end{aligned}
$$

i.e. as homomorphisms from the Abelian sub-algebra $\mathcal{C}^{2}(\mathbb{R}) \otimes 1$ of $\mathcal{B}\left(L^{2}(\mathbb{R}) \otimes\right.$ $\left.L^{2}(\Omega, \mathcal{F}, P)\right)$ into $\mathcal{B}\left(L^{2}(\mathbb{R}) \otimes L^{2}(\Omega, \mathcal{F}, P)\right)$. These homomorphisms $j_{t}$ are characterized by the property of being the unique solution of the stochastic equation (30) with initial condition

$$
j_{0}(f)=f
$$

where we use the same symbol $f$ to denote the function $f$ and the multiplication operator by the function $f$ acting on $L^{2}(\mathbb{R})$.

Now notice that the SDE (30) continues to have a meaning even if the multiplication operator by the function $f$ is replaced by an arbitrary operator $x$ acting on $L^{2}(\mathbb{R})$ :

$$
d j_{t}(x)=i\left[p(a), j_{t}(x)\right] d W_{t}-\frac{1}{2}\left[p,(a),\left[p(a), j_{t}(x)\right]\right] d t+i\left[p\left(l_{1}\right), j_{t}(x)\right] d t
$$


and the initial condition by its natural generalization

$$
j_{0}(x)=x
$$

Under general conditions one can extend the existence and uniqueness theorem for the classical equation to the quantum equation, for example by proving the convergence of the iterated series in an appropriate topology.

Then, by a standard application of the classical Itô table, we can conclude that

$$
j_{t}(x) j_{t}(y)=j_{t}(x y)
$$

Similarly, using again uniqueness and the identity

$$
[a, b]^{*}=\left[b^{*}, a^{*}\right]
$$

one proves that

$$
j_{t}(x)^{*}=j_{t}\left(x^{*}\right)
$$

and therefore $j_{t}$ is a $*$-homomorphism from $\mathcal{B}\left(L^{2}(\mathbb{R})\right) \equiv \mathcal{B}\left(L^{2}(\mathbb{R})\right) \otimes 1$ to $\mathcal{B}\left(L^{2}(\mathbb{R})\right) \otimes \mathcal{B}\left(L^{2}(\Omega, \mathcal{F}, P)\right)$.

To complete the analogy with Koopman's argument we ask ourselves the following question: does there exist a 1-parameter family of linear operators $U_{t}$ acting on $L^{2}(\mathbb{R}) \otimes L^{2}$ (Wiener space) such that, for any random variable $X_{0}$, independent of $\left(W_{t}\right)$ and with initial distribution absolutely continuous with respect to the Lebesgue measure on $\mathbb{R}$, and for any $f \in L^{\infty}(\mathbb{R})$, one has

$$
U_{t} M_{f\left(X_{0}\right)} U_{t}^{*}=M_{f\left(X_{t}\right)}
$$

Notice that, if such an $U_{t}$ exist, then it must be unitary because:

(i) $U_{t} U_{t}^{*}=1 \Leftrightarrow j_{t}(1)=1$

(ii) $U_{t}^{*} U_{t}=1 \Leftrightarrow$ the map $f \mapsto U_{t} M_{f\left(X_{0}\right)} U_{t}^{*}$ is a $*$-homomorphism

To answer the above question one can argue as follows: if such $U_{t}$ exists it must be a functional of the $\left(W_{s}\right)_{s \leq t}$ because of (31). Thus we postulate an equation for $U_{t}$ of the form

$$
d U_{t}=\left(\alpha d t+\beta d W_{t}\right) U_{t}
$$

Then, differentiating the unitarity conditions for $U_{t}$, i.e. $U_{t} U_{t}^{*}=U_{t}^{*} U_{t}=1$ and using the classical Itô table, we deduce a relation between $\alpha$ and $\beta$. After that we differentiate, again using the classical Itô table, both sides of the identity

$$
f\left(X_{t}\right)=j_{t}\left(M_{f}\right)=U_{t} M_{f}\left(X_{0}\right) U_{t}^{*}
$$

and identify the coefficients of the differentials $d W_{t}$ and $d t$. The result is a classical,i.e. driven by classical BM, stochastic Schrödinger equation (SSE):

$$
d U_{t}=\left(-i K d W_{t}-\left[\frac{1}{2} K^{2}+i H\right] d t\right) U_{t}
$$


with (in the notations (28), (29):

$$
H=H^{*}=-p\left(l_{1}\right) \quad ; \quad K=K^{*}=-p(a)
$$

In absence of noise, i.e. $K=0(\Leftrightarrow a=0$ in (26)), equation (33) becomes an usual Schrödinger equation (21). Notice that both $H$ and $K$ are symmetric hence, without the dissipation term $K^{2} / 2$, equation (33) would be formally Hamiltonian. However the dissipation term is essential for the unitarity of the solution. Thus we see that, within the context of stochastic differential equations, the requirement of unitarity of the solution is in contradiction with the Hamiltonian character of the equation. This problem will persist in the context of quantum stochastic differential equations: only the white noise approach will be able to overcome this problem.

\section{Quantum stochastic Schrödinger and Heisenberg equations}

The transition from classical to quantum stochastic Schrödinger and Heisenberg equations is now accomplished by using the quantum decomposition of the classical Brownian motion $d W_{t}=d B_{t}^{+}+d B_{t}$ and allowing for different coefficients of the quantum stochastic differentials $d B_{t}^{+}$and $d B_{t}$.

Differentiating the unitarity conditions for $U_{t}$ and using the HudsonParthasaraty Ito table, we deduce a relation between the coefficients of $d B_{t}^{+}$, $d B_{t}$ and $d t$. The final form of the equation is then:

$$
d U_{t}=\left(D d B_{t}^{+}-D^{+} d B_{t}-\left[\frac{1}{2} D^{+} D+i H\right] d t\right) U_{t}
$$

where $D$ and $H$ are arbitrary, say bounded, operators and $H=H^{*}$. The same argument, applied to a more general equation, including also the number differential $d N_{t}$ leads to the most general Hudson-Parthasaraty stochastic Schrödinger equation:

$$
d U_{t}=\left(S D d B_{t}^{+}-D^{*} d B_{t}+(S-1) d N_{t}+\left(-\frac{1}{2} D^{+} D+i H\right) d t\right) U_{t}
$$

where $D$ and $H$ are as above and $S$ must be a unitary operator. Notice that, contrarily to the diffusion case $(34)$, here the Hamiltonian nature of the equation is lost even at the level of the martingale term: the non Hamiltonian nature of equation (35) is not due only to the presence of the dissipative term $D^{+} D / 2$ but also of the unitary operator $S$. The deep meaning of this apparently strange structure can only be understood in terms of quantum white noise calculus (see section (13) below). 


\section{White Noise Schrödinger and Heisenberg equations}

The white noise equations live on spaces of the form

$$
\mathcal{H}=\mathcal{H}_{S} \otimes \Gamma
$$

where the Hilbert space $\mathcal{H}_{S}$ is called the initial (or system) space, and the Hilbert space $\Gamma$ is called the noise space.

For 1 -st order white noise equations the typical $\Gamma$ is the same as for Hudson-Parthasarathy equations, i.e. a Fock space over a 1-particle space of the form $L^{2}(\mathbb{R} ; \mathcal{K})$ where $\mathcal{K}$ is another Hilbert space, called the multiplicity space (in mathematics) or polarization space (in physics). A WN Schrödinger (or Hamiltonian) equation is an equation of the form

$$
\partial_{t} U_{t}=-i H_{t} U_{t} \quad ; \quad U_{0}=1
$$

where $H_{t}=H_{t}^{*}$ is a symmetric functional of white noise and the associated Heisenberg equation (from now on we will consider only the inner case)

$$
\partial_{t} X_{t}=-i\left[H_{t}, X_{t}\right] \quad ; \quad X_{0}=X \in \mathcal{B}(\mathcal{H})
$$

Since in the inner case, as explained in section (7), the solution of the Heisenberg equation has the form

$$
X_{t}=U_{t} X_{t} U_{t}^{*}
$$

it will be sufficient to consider the Schrödinger equation

\section{Stochastic equations associated to $1-$ st order WN Schrödinger equations}

The simplest WN equations are the 1-st order WN Schrödinger equations, for which $H_{t}$ has the form:

$$
H_{t}=D b_{t}^{+}+D^{+} b_{t}+T b_{t}^{+} b_{t}+C=D \otimes b_{t}^{+}+\ldots
$$

Notice that the right hand side is formally symmetric if

$$
T^{+}=T \quad ; \quad C^{+}=C
$$

Diffusion WN equations are characterized by the condition:

$$
T=0
$$

Example.

$$
\partial_{t} U_{t}=-i H_{t} U_{t}=-i\left(D b_{t}^{+}+D^{+} b_{t}\right) U_{t}
$$


if $D=D^{+}$this becomes

$$
\partial_{t} U_{t}=-i H_{t} U_{t}=-i D\left(b_{t}^{+}+b_{t}\right) U_{t}=-i D w_{t} U_{t}
$$

in terms of Brownian motion

$$
\frac{d}{d t} U_{t}=-i D \frac{d}{d t} W_{t} U_{t}
$$

Warning: in section (4) one might be tempted to use the naive relation

$$
\frac{d}{d t} W_{t}=w_{t} \Leftrightarrow d W_{t}=w_{t} d t
$$

and to conclude that the classical WN equation (37) is equivalent to the classical stochastic differential equation

$$
d U_{t}=-i D d W_{t} U_{t}
$$

but this would lead to a contradiction because it can be proved that equation (39), does not admit any unitary solution while WN Hamiltonian equations of the form (36) can be shown to admit unitary solutions.

In fact it is true that WNH equations of the form (36) are canonically associated to stochastic differential equations but, for the determination of this stochastic equation, the naive prescription (38) is not sufficient and a much subtler rule must be used. The correct answer is given by the following theorem.

Theorem 5. Let $A, C$ and $T=T^{*}$ be bounded operators on the initial space $\mathcal{H}_{S}$. Then the white noise Schrödinger equation

$$
\partial_{t} U_{t}=-i\left(A b_{t}+A^{*} b_{t}^{+}+b_{t}^{+} T b_{t}+C\right) U_{t} \quad ; U_{0}=1
$$

$\left(T=T^{*} ; C=C^{*}\right)$ is equivalent to the following stochastic differential equation

$$
\begin{aligned}
d U_{t} & =\left(S D d B_{t}^{+}-D^{*} d B_{t}\right. \\
& \left.+\frac{1}{2 \operatorname{Re}\left(\gamma_{-}\right)}(S-1) d N_{t}+\left(-\gamma_{-} D^{+} D+i\left|\gamma_{-}\right|^{2} D^{+} T D-i C\right) d t\right) U_{t}
\end{aligned}
$$

where the unitary operator

$$
S:=\frac{1-i T}{1+i T}
$$

is the Cayley transform of $T$ and:

$$
D^{+}:=i A \frac{1}{1+i T}
$$


Remark. The two equations can be interpreted in the weak sense on the total domain of extended number vectors with continuous test functions (the vectors of the form $\xi_{S} \otimes n$ with $\xi_{S} \in \mathcal{H}_{S}$ and $n$ a number vector with continuous test functions).

Once taken the matrix elements of both sides in a pair of such vectors both equations become (numerical) ordinary differential equations. Equivalence here means that the solutions of these two equations coincide.

The notion of weak solution of an equation is well established both in classical and stochastic analysis. Much stronger notions can be introduced in the present situation but, for the purpose of comparing the meaning and the solutions of the equations (40), (41), this is sufficient.

The transition from (40) to (41) is achieved through the operation of causal normal order which is also responsible for the emergence of the (complex) constant $\gamma_{-}$. This constant (and its generalizations to more complex systems) have a fundamental importance in physics (where they are interpreted as generalized susceptivities) but, in a purely mathematical treatment, they can be absorbed in the notations.

The proof of the equivalence is based on one of the deepest analytical principles emerged from the stochastic limit of quantum theory, the time consecutive principle, whose proof is based on another mathematical development motivated by the same theory, the theory of distributions on the standard simplex. These topics will not be discussed here. For additional information the reader is referred to the references mentioned in section (2).

The most general unitary stochastic differential equation (35), in the sense of Hudson-Parthasarathy, is obtained from (41) by putting $\gamma_{-}=\frac{1}{2}$ and by the change of notation $\left|\gamma_{-}\right|^{2} D^{+} T D-C=: H$

From formulae (42), (43), we can now understand in what sense the white noise approach unveils the microscopic structure of the coefficients of the unitary equations in the Hudson-Parthasarathy sense (i.e. (35)): the fact that only the special combination of coefficients of equation (35) can give a unitary solution is the result of a calculation based on the quantum Ito table, but the meaning of these conditions is quite obscure. On the contrary, looking at (40) we see that the unitarity condition is equivalent to the formal symmetry of the WH Hamiltonian. The analogue equivalence between white noise Heisenberg and stochastic Heisenberg equation has been recently established in [AcAyOu05] and, in its Hilbert module formulation [AcAyOu05], it leads to a purely algebraic stochastic generalization of the derivation condition (24) (an analytical generalization of this condition, based on operator valued measures and mutual quadratic variations was discussed in [AcHud89]).

\section{The Renormalized Square of classical WN}

We have seen that the quantum decomposition of the 1-st order classical WN is: 


$$
w_{t}=b_{t}^{+}+b_{t}
$$

If one tries to do the square of $w_{t}$ naively, one obtains:

$$
w_{t}^{2}=\left(b_{t}^{+}+b_{t}\right)^{2}=b_{t}^{+2}+b_{t}^{2}+b_{t}^{+} b_{t}+b_{t} b_{t}^{+}=b_{t}^{+2}+b_{t}^{2}+2 b_{t}^{+} b_{t}+\delta(0)
$$

where in the last identity we have applied the commutation relations (7) to the case $t=s$. This application is purely formal because $\delta(t-s)$ is a distribution and expressions like $\delta(0)$ are meaningless. The standard procedure to overcome this problem is to subtract the diverging quantity $\delta(0)$ (additive renormalization) and to conjecture that the result i.e.:

$$
: w_{t}^{2}:=b_{t}^{+2}+b_{t}^{2}+2 b_{t}^{+} b_{t}
$$

is, up to a constant, the quantum decomposition of the square of the classical white noise.

However, even after this renormalization the right hand side of (45) is ill defined. The problem is that, as will be shown in the following session, expressions like $b_{t}^{+2}, b_{t}^{2}$ are not well defined even as operator valued distributions!

\section{Basic new idea: renormalize the commutation relations}

The problem of giving a meaning to expressions like $b_{t}^{2}, b_{t}^{+2}$ has its origins in the fact that the commutation relations

$$
\left[b_{s}, b_{t}^{+}\right]=\delta(t-s)
$$

imply that

$$
\left[b_{s}^{2}, b_{t}^{+2}\right]=4 \delta(t-s) b_{s}^{+} b_{t}+2 \delta(t-s)^{2}
$$

But what does it mean $\delta(t-s)^{2}$ ? We found in the literature [Ivanov79] (see also [BogLogTod69] and [Vlad66]) the following prescription: On an appropriate test function space the following identity holds

$$
\delta(t)^{2}=c \delta(t)
$$

where the constant $c \in \mathbb{C}$ is arbitrary. (A poof of this statement and the description of the test function space can be found in [AcLuVo99].)

Using this prescription in (46) we obtain the renormalized commutation relations:

$$
\left[b_{s}^{2}, b_{t}^{+2}\right]=4 \delta(t-s) b_{s}^{+} b_{t}+2 c \delta(t-s)
$$

Moreover (without any renormalization!)

$$
\left[b_{s}^{2}, b_{t}^{+} b_{t}\right]=2 \delta(t-s) b_{t}^{2}
$$


From (47) and (48) it follows that, after renormalization, the self-adjoint set of operators

$$
b_{s}^{2}, b_{s}^{+2}, b_{t}^{+} b_{t}, c=(\text { central element })
$$

is closed under commutators, i.e. the linear span of these operators is a $*-$ Lie algebra.

In test function language the renormalized square of white noise $*$-Lie algebra can be defined as follows

$$
\begin{gathered}
b_{\varphi}^{+}=\int d t \varphi(t) b_{t}^{+2} ; \quad b_{\varphi}=\left(b_{\varphi}^{+}\right)^{+} \\
n_{\varphi}=\int d t \varphi(t) b_{t}^{+} b_{t}
\end{gathered}
$$

where $\varphi \in \mathcal{S}$ and $\mathcal{S}$ is a space of test functions (for example one can choose the finitely valued step functions on $\mathbb{R}$ ). This leads to the commutation relations:

$$
\begin{gathered}
{\left[b_{\varphi}, b_{\psi}^{+}\right]=\gamma\langle\varphi, \psi\rangle+n_{\bar{\varphi} \psi}} \\
{\left[n_{\varphi}, b_{\psi}\right]=-2 b_{\bar{\varphi} \psi}} \\
{\left[n_{\varphi}, b_{\psi}^{+}\right]=2 b_{\varphi \psi}^{+}} \\
\left(b_{\varphi}^{+}\right)^{+}=b_{\varphi} \quad ; \quad n_{\varphi}^{+}=n_{\bar{\varphi}}
\end{gathered}
$$

Definition A Fock representation of the $*$-Lie algebra of the RSWN is a representation of this $*$-Lie algebra as operators on a Hilbert space $\mathcal{H}$ with a unit vector $\Phi$ satisfying the following conditions (for notational simplicity we will use the same symbol for an element of the $*$-Lie algebra of the RSWN and for its image acting on $\mathcal{H}$ ).

(i) The set of vectors (called the quadratic number vectors)

$$
\left\{\left(b_{\varphi}^{+}\right)^{n} \Phi: n \in \mathbb{N}, \varphi \in \mathcal{S}\right\}
$$

is well defined and total in $\mathcal{H}$.

(ii) The algebraic linear span of the quadratic number vectors is invariant under the action of the operators $\left\{b_{\varphi}^{+}, b_{\varphi}, n_{\varphi}: \varphi \in \mathcal{S}\right\}$.

(iii) The commutation relations (49), (50), (51), (52) take place on the quadratic number vectors

(iv) The Fock property holds:

$$
b_{\varphi} \Phi=n_{\varphi} \Phi=0
$$




\section{Existence of Fock representations}

Having defined the Fock representation the first problem is its existence. In case of the first order white noise this is a well known result since the early days of quantum theory.

Theorem (Fock 1930) The Fock representation of the first order white noise (i.e. the current algebra over $\mathbb{R}$ of the CCR Lie algebra $\left[a, a^{+}\right]=1$, for the notion of current algebra see section (18)) exists and is unique up to unitary isomorphism.

The analogue for the RSWN Lie algebra was established more recently. Theorem (Accardi , Lu, Volovich 1999) The Fock representation of the second order white noise (current algebra over $\mathbb{R}$ of the Lie algebra $\operatorname{sl}(2, \mathbb{R})$ ) exists and is unique up to unitary isomorphism.

A direct proof of this result is a nontrivial application of the principle that algebra implies statistics, described in its simplest form in Section (3): one proves that, if the required Fock representation exists, then the scalar product of two number vectors is uniquely determined by the commutation relations (49), (50), (51), (52) and the Fock property (53). Then, and this is the difficult part, one has to prove that this is indeed a scalar product, i.e. that it is positive definite (cf. [AcLuVo99]).

In section (21) we will come back to this point. Before that let us analyze some consequences of the above theorem. More precisely let us apply to this case the basic general principle of QP discussed in section (3): algebra implies statistics. In section (3) we have seen that the application of this principle to the first order white noise shows that the corresponding algebra implies Gaussian and Poisson statistics. It is therefore natural to rise the following question:

Which statistics is implied by the algebra of the renormalized Square of $W N$ ?

The answer to this question was given by Accardi, Franz and Skeide in the paper [AcFrSk00].

\section{Classical subprocesses associated to the second order white noise}

To understand this answer it is convenient to take as starting point the analogy with the q-decomposition of the compensated classical Poisson process with intensity $\beta^{-1}$

$$
\dot{p}_{t}=b_{t}^{+}+b_{t}+\beta b_{t}^{+} b_{t}
$$

At the end of section (5) we have seen that $\beta=0$ is the only critical case and corresponds to the transition from classical scalar valued standard compensated Poisson process with intensity $\beta^{-1}$. 
This analysis is extended in the paper [AcFrSk00] to the renormalized square of white noise by considering the classical subprocesses

$$
X_{\beta}(t):=b_{t}^{+2}+b_{t}^{2}+\beta b_{t}^{+} b_{t}
$$

where $\beta$ is a real number. It is then proved that now there are 2 critical values of $\beta$, namely:

$$
\beta= \pm 2
$$

The value +2 corresponding to the renormalized square of the position (classical) white noise, i.e.

$$
\begin{aligned}
& w_{t}^{2}=\left|b_{t}^{+}+b_{t}\right|^{2}=b_{t}^{+2}+b_{t}^{2}+b_{t}^{+} b_{t}+b_{t} b_{t}^{+}= \\
= & b_{t}^{+2}+b_{t}^{2}+2 b_{t}^{+} b_{t}+\delta(0) \equiv b_{t}^{+2}+b_{t}^{2}+2 b_{t}^{+} b_{t}
\end{aligned}
$$

and the value -2 to the renormalized square of the momentum white noise, i.e.

$$
\left(b_{t}^{+}-b_{t}\right) / i
$$

The vacuum distribution of both processes is the Gamma-process

$$
\mu(d x)=\frac{|x|^{m_{0}-1}}{\Gamma\left(m_{0}\right)} e^{-\beta x} \chi_{\beta \mathbb{R}_{+}}
$$

whose parameter $m_{0}>0$ is uniquely determined by the choice of the unitary representation of $S L(2, \mathbb{R})$ corresponding to the representation of the SWN algebra (cf. [ACFRSK00]).

In this functional realization the number vectors become the Laguerre polynomials which are orthogonal for the Gamma distribution.

Since the Gamma-distributions are precisely the distributions of the $\chi^{2}$ random variables, this result confirms the naive intuition that the distribution of the [renormalized] square of white noise should be a Gamma-distributions.

For $|\beta|<2$ the intensity of the jumps is not strong enough and each of the classical random variables

$$
X_{\beta}(t):=b_{t}^{+2}+b_{t}^{2}+\beta b_{t}^{+} b_{t}
$$

still has a density whose explicit form, in terms of the $\Gamma$-function is:

$$
\mu(d x)=C \exp \left(-\frac{(2 \arccos \beta+\pi) x}{2 \sqrt{1-\beta^{2}}}\right)\left|\Gamma\left(\frac{m_{0}}{2}+\frac{i x}{2 \sqrt{1-\beta^{2}}}\right)\right|^{2}
$$

( $C$ is a normalization constant). The corresponding orthogonal polynomials are the Meixner-Pollaczek polynomials. For $m_{0}$ integer more explicit formulae for these densities were found by Grigelionis [Grig01],[Grig99], [Grig00c]:

$$
|\Gamma(n+i x)|^{2}=\frac{\pi x\left(1+x^{2}\right) \ldots\left((n-1)^{2}+x^{2}\right)}{\sinh (\pi x)} ; n=1,2, \ldots, \quad, x \in \mathbb{R}
$$




$$
\begin{gathered}
\left|\Gamma\left(\frac{1}{2}+i x\right)\right|^{2}=\frac{\pi}{\cosh (\pi x)} \quad, \quad x \in \mathbb{R} \\
\left|\Gamma\left(n+\frac{1}{2}+i x\right)\right|^{2} \\
=\frac{\pi\left(\frac{1}{4}+x^{2}\right) \ldots\left((n-1) n+\frac{1}{4}+x^{2}\right)}{\cosh (\pi x)} \quad, \quad n=1,2, \ldots ; x \in \mathbb{R}
\end{gathered}
$$

Finally, for $|\beta|>2$ the jumps dominate and the classical random variable

$$
X_{\beta}(t):=b_{t}^{+2}+b_{t}^{2}+\beta b_{t}^{+} b_{t}
$$

has a discrete vacuum distribution i.e. the negative binomial (Pascal) distribution which plays, for the square of white noise the a role analogue to the one played by the geometric (or Gibbs) distribution for the first order white noise:

$$
\mu=C \sum_{n=0}^{\infty} \frac{c^{2 n}\left(m_{0}\right)_{n}}{n !} \delta_{\operatorname{sgn}\left(\beta\left((c-1 / c)\left(n+m_{0} / 2\right)\right)\right.}
$$

where $\left(m_{0}\right)_{n}$ denotes the Pochammer symbol,

$$
\left(m_{0}\right)_{n}=m_{0}\left(m_{0}+1\right) \cdots\left(m_{0}+n-1\right)
$$

and

$$
C^{-1}=\sum_{n=0}^{\infty} \frac{c^{2 n}\left(m_{0}\right)_{n}}{n !}=\left(1-c^{2}\right)^{-m_{0}}
$$

More precisely, if $\beta>0$

$$
\begin{gathered}
P_{n}(x)=(-1)^{n} \prod_{k=1}^{n} \frac{n+m_{0}-1}{n} M_{n}\left(\frac{x}{c-1 / c}-\frac{m_{0}}{2} ; m_{0} ; c^{2}\right) \\
\prod_{k=1}^{n} \frac{n+m_{0}-1}{n} M_{n}\left(-\frac{x}{c-1 / c}+\frac{m_{0}}{2} ; m_{0} ; c^{2}\right)
\end{gathered}
$$

where the $M_{n}$ are the Meixner polynomials and

$$
c=\beta-\sqrt{\beta^{2}-4}
$$

if $\beta>+2$, while if $\beta<-2$ then:

$$
c=-\beta-\sqrt{\beta^{2}-4}
$$

In conclusion: the 1-parameter family (54) of vacuum operator processes gives rise, according to the values of the parameter, to three classes of processes: 
$|\lambda|=2$ classical Gamma processes (Laguerre polynomials)

$|\lambda|<2$ Meixner processes (Meixner-Pollaczek polynomials)

$|\lambda|>2$ negative binomial (Pascal) processes (Meixner polynomials)

These three classes of stochastic processes exactly coincide with the remaining three Meixner classes! Comparing this result with the remark at the end of section (5) we see that the first and second powers of white noise account exactly for the five Meixner classes.

These 5 classes were known since 1934 (date of publication of Meixner's paper), but only through their quantum decomposition was their common structure made clear. Moreover the infinite divisibility of these distributions, which is not easy to prove analytically, now became an easy corollary of the general phenomenon described in section (19) below.

Since the 1-st and second powers of white noise gave rise, in the sense just explained, tot he five Meixner classes, it is quite natural to ask oneself what happens with the higher powers of WN. In order to answer this question we have to recall the connection between additive independent increment processes and current algebras over Lie algebras.

\section{Current representations of Lie algebras}

Intuitively, if $\{\mathcal{L},[\cdot, \cdot], *\}$ is a $*$-Lie algebra, a current algebra of $\mathcal{L}$ over $\mathbb{R}^{d}$ is a vector space $\mathcal{T}$ of $\mathcal{L}$-valued functions defined on $\mathbb{R}^{d}$ and closed under the pointwise operations:

$$
[\varphi, \psi](t):=[\varphi(t), \psi(t)] ; \quad \varphi^{*}(t):=\varphi(t)^{*} \quad ; t \in \mathbb{R}, \varphi \in \mathcal{T}
$$

For example, if $X_{1}, \ldots, X_{k}$ are generators of $\mathcal{L}$ one can fix a space $\mathcal{S}$, of complex valued test functions on $\mathbb{R}$ and to each $\varphi \in \mathcal{S}$ and $j \in\{1, \ldots, k\}$ one can associate the $\mathcal{L}$-valued function on $\mathbb{R} X_{j}(\varphi)$ defined by:

$$
X_{j}(\varphi)(t):=\varphi(t) X_{j} ; \quad t \in \mathbb{R}
$$

Definition 6. Let $\mathcal{G}$ be a complex $*-$ Lie algebra. A (canonical) set of generators of $\mathcal{G}$ is a linear basis of $\mathcal{G}$

$$
l_{\alpha}^{+}, l_{\alpha}^{-}, l_{\beta}^{0} \quad, \alpha \in I, \quad \beta \in I_{0}
$$

where $I_{0}, I$ are sets, satisfying the following conditions:

$$
\begin{array}{ll}
\left(l_{\beta}^{0}\right)^{*}=l_{\beta}^{0} & ; \quad \forall \beta \in I_{0} \\
\left(l_{\alpha}^{+}\right)^{*}=l_{\alpha}^{-} & ; \quad \forall \alpha \in I
\end{array}
$$

and all the central elements among the generators are of $l^{0}$-type (i.e. selfadjoint). 
We will denote $c_{\alpha \beta}^{\gamma}\left(\varepsilon, \varepsilon^{\prime}, \delta\right)$ the structure constants of $\mathcal{G}$ with respect to the generators $\left(l_{\alpha}^{\varepsilon}\right)$, i.e., with $\alpha, \beta \in I \cup I_{0}, \varepsilon, \varepsilon^{\prime}, \delta \in\{+,-, 0\}$, and, assuming summation over repeated indices:

$$
\begin{gathered}
{\left[l_{\alpha}^{\varepsilon}, l_{\beta}^{\varepsilon^{\prime}}\right]=c_{\alpha \beta}^{\gamma}\left(\varepsilon, \varepsilon^{\prime}, \delta\right) l_{\gamma}^{\delta}=} \\
:=\sum_{\gamma \in I_{0}} c_{\alpha \beta}^{\gamma}\left(\varepsilon, \varepsilon^{\prime}, 0\right) l_{\gamma}^{0}+\sum_{\gamma \in I} c_{\alpha \beta}^{\gamma}\left(\varepsilon, \varepsilon^{\prime},+\right) l_{\gamma}^{+}+\sum_{\gamma \in I} c_{\alpha \beta}^{\gamma}\left(\varepsilon, \varepsilon^{\prime},-\right) l_{\gamma}^{-}
\end{gathered}
$$

In the following we will consider only locally finite Lie algebras, i.e. those such that, for any pair $\alpha, \beta \in I \cup I_{0}$ only a finite number of the structure constants $c_{\alpha \beta}^{\gamma}\left(\varepsilon, \varepsilon^{\prime}, \delta\right)$ is different from zero.

Definition 7. Let be given:

- $a *$-Lie algebra $\mathcal{G}$

- a measurable space $(S, \mathcal{B})$

- a*-sub-algebra $\mathcal{C} \subseteq L_{\mathbb{C}}^{\infty}(S, \mathcal{B})$ for the pointwise operations.

The current algebra of $\mathcal{G}$ over $\mathcal{C}$ is the $*$-Lie algebra

$$
\mathcal{G}(\mathcal{C}):=\{\mathcal{C} \otimes \mathcal{G},[\cdot, \cdot]\}
$$

where $\mathcal{C} \otimes \mathcal{G}$ is the algebraic tensor product, the Lie brackets $[\cdot, \cdot \cdot]$ are given by

$$
\left[f \otimes l, g \otimes l^{\prime}\right]:=f g \otimes\left[l, l^{\prime}\right] \quad ; \quad f, g \in \mathcal{C}, l, l^{\prime} \in \mathcal{G}
$$

and the involution $*$ is given by

$$
(f \otimes l)^{*}:=\bar{f} \otimes l^{*} \quad ; \quad f \in \mathcal{C}, l \in \mathcal{G}
$$

where $\bar{f}$ denotes complex conjugate. In the following we shall use the notation:

$$
l(f):=f \otimes l \quad ; \quad f, g \in \mathcal{C}, l, \in \mathcal{G}, f \in \mathcal{C}
$$

Remark. If $\left(l_{\gamma}^{\varepsilon}\right)$ is a (canonical) set of generators of $\mathcal{G}$ then the set

$$
\left\{f \otimes l_{\alpha}^{+}, f^{*} \otimes l_{\alpha}^{-}, R e(f) \otimes l_{\gamma}^{0}: \alpha \in I, \gamma \in I_{0}, f \in \mathcal{C}\right\}
$$

$(\operatorname{Re}(f):=(f+\bar{f}) / 2)$ is a (canonical) set of generators of $\mathcal{G}(\mathcal{C})$.

In the following we will use the notations:

$$
l_{\alpha}^{+}(f):=f \otimes l_{\alpha}^{+} \quad, \quad l_{\alpha}^{-}(f):=f^{*} \otimes l_{\alpha}^{-} \quad, \quad l_{\alpha}^{0}(f):=f \otimes l_{\gamma}^{0}
$$

and, when no confusion can arise, we will often speak of the current algebra $\left(l_{\alpha}^{\varepsilon}(f)\right)$.

Definition 8. A representation of a $*$-Lie algebra $\mathcal{G}$ is a triple:

$$
\{\mathcal{H}, \mathcal{D}, \pi\}
$$


where

- $\mathcal{H}$ is an Hilbert space

- $\mathcal{D}$ is a total subset of $\mathcal{H}$

$-\pi: \mathcal{D} \rightarrow \mathcal{H}$ is a map such that:

(i) for any $l \in \mathcal{G}, \pi(l)$ is a pre-closed operator on $\mathcal{D}$ with adjoint $\pi\left(l^{*}\right)$

(ii) for any $l, l^{\prime} \in \mathcal{G}$,

$$
\pi\left(\left[l, l^{\prime}\right]\right)=\left[\pi(l), \pi\left(l^{\prime}\right)\right]
$$

where the commutator on the right hand side is meant weakly on $\mathcal{D}$.

Remark. At the algebraic level the existence of representations of current algebras of an arbitrary pair $\left\{\mathcal{G},\left(l_{\alpha}^{\varepsilon}\right)\right\}$ over an arbitrary measure space $(S, \mathcal{B}, \mu)$ and sub-*-algebra

$$
\mathcal{C} \subseteq L^{\infty}(S, \mathcal{B}, \mu)
$$

is easily established. In fact, if $\{\pi, \mathcal{K}\}$ is any representation of $\mathcal{G}$ one can define a structure of $*$-Lie algebra on

$$
\mathcal{C} \otimes \pi(\mathcal{G}) \in \mathcal{L}\left(L^{2}(S, \mathcal{B}, \mu) \otimes \mathcal{K}\right)
$$

in terms of the brackets

$$
\left[f \otimes \pi(e), g \otimes \pi\left(l^{\prime}\right)\right]:=f g \otimes \pi\left(\left[l, l^{\prime}\right]\right)
$$

Therefore, defining:

$$
\begin{aligned}
& l_{\alpha}^{+}(f):=f \otimes \pi\left(l_{\alpha}^{+}\right) \\
& l_{\alpha}^{\circ}(f):=f \otimes \pi\left(l_{\alpha}^{\circ}\right)
\end{aligned}
$$

one has

$$
\begin{gathered}
l_{\alpha}^{+}(f)^{*}=\bar{f} \otimes \pi\left(l_{\alpha}^{+}\right)^{*}=\bar{f} \otimes \pi\left(\left(l_{\alpha}^{+}\right)^{*}\right)=\bar{f} \otimes \pi\left(l_{\alpha}^{-}\right) \\
\left(l_{\beta}^{0}(f)\right)^{*}=\bar{f} \otimes \pi\left(l_{\beta}^{0}\right)^{*}=\bar{f} \otimes \pi\left(l_{\beta}^{0}\right)=l_{\beta}^{0}(\bar{f}) \\
{\left[l_{\alpha}^{\varepsilon}(f), l_{\beta}^{\varepsilon^{\prime}}(g)\right]=\left[f^{\varepsilon} \otimes \pi\left(l_{\alpha}^{\varepsilon}\right), g^{\varepsilon^{\prime}} \otimes \pi\left(l_{\beta}^{\varepsilon^{\prime}}\right)\right]=f^{\varepsilon} g^{\varepsilon^{\prime}} \otimes\left[\pi\left(l_{\alpha}^{\varepsilon}\right), \pi\left(l_{\beta}^{\varepsilon^{\prime}}\right)\right]} \\
=f^{\varepsilon} g^{\varepsilon^{\prime}} \otimes \pi\left[l_{\alpha}^{\varepsilon}, l_{\beta}^{\varepsilon^{\prime}}\right]=c_{\alpha \beta}^{\gamma}\left(\varepsilon, \varepsilon^{\prime}, \delta\right) f^{\varepsilon} g^{\varepsilon^{\prime}} \otimes l_{\gamma}^{\delta}=c_{\alpha \beta}^{\gamma}\left(\varepsilon, \varepsilon^{\prime}, \delta\right) l_{\gamma}^{\delta}\left(f^{\varepsilon} g^{\varepsilon^{\prime}}\right)
\end{gathered}
$$

Thus the current algebra relations are verified. This proves that any representation of $\mathcal{G}$ can be lifted to a representation of the current algebra $\mathcal{G} \otimes \mathcal{G}$.

Definition 9. Let $\mathcal{G}$ be a*-Lie algebra with a (canonical) set of generators $\left(l_{\alpha}^{\varepsilon}\right)$. A representation $\{\mathcal{K}, \mathcal{D}, \pi\}$ of $\mathcal{G}$ is called weakly irreducible if the images of the central elements are multiples of the identity.

$A$ representation $\{\mathcal{K}, \mathcal{D}, \pi\}$ of $\mathcal{G}$ on a Hilbert space $\mathcal{K}$ is called a Fock representation if it is weakly irreducible and: 
(i) there exists a unit vector $\Phi \in \mathcal{K}$ such that $\forall \alpha \in I$ and $\forall \beta \in I_{0}$, with the exception of those $\beta \in I_{0}$ which correspond to central elements, one has

$$
\pi\left(l_{\alpha}^{-}\right) \Phi=\pi\left(l_{\beta}^{0}\right) \Phi=0
$$

(ii) the set

$$
\left\{\pi\left(l_{\alpha}^{+}\right)^{n} \Phi: \alpha \in I, n \in \mathbb{N}\right\}
$$

is total in $\mathcal{K}$.

All the difficulties found in the construction of a coherent theory of higher powers of white noise can be summarized in the following problem.

Problem. Let $\left\{\mathcal{G},\left(l_{\alpha}^{\varepsilon}\right)\right\}$ be a $*-$ Lie algebra with a set of generators. Suppose that $\left\{\mathcal{G},\left(l_{\alpha}^{\varepsilon}\right)\right\}$ admits a Fock representation. Under which conditions on the measure space $(S, \mathcal{B}, \mu)$ and on the $*$-sub-algebra

$$
\mathcal{C} \subseteq L^{\infty}(S, \mathcal{B}, \mu)
$$

does the current algebra

$$
\left\{l_{\alpha}^{\varepsilon}(f): \varepsilon \in\{+,-, 0\}, \quad \alpha \in I \text { or } \alpha \in I_{0}, \quad f \in \mathcal{C}\right\}
$$

admit a Fock representation?

Example (1) The Heisenberg algebra is the $*$-Lie algebra with set of generators $\left\{a^{+}, a, 1\right\}$ ( 1 here and in the following denotes the central element) and relations

$$
\left[a, a^{+}\right]=1
$$

The associated current algebra over $\mathbb{R}^{d}$ with Lebesgue measure admits a Fock representation which is the standard $d$-dimensional white noise or the free boson Fock field over $\mathbb{R}^{d}$.

Example (2) The oscillator algebra is the $*$-Lie algebra with set of generators $\left\{a^{+}, a, a^{+} a, 1\right\}$ and relations deduced from (57). The associated current algebra over $\mathbb{R}^{d}$ with Lebesgue measure admits a Fock representation which can be called the Hudson-Parthasarathy algebra over $\mathbb{R}^{d}$.

Example (3) The square-oscillator algebra is the $*$-Lie algebra with set of generators $\left\{a^{+2}, a^{2}, a^{+} a, 1\right\}$ and relations deduced from (57). The associated current algebra over $\mathbb{R}^{d}$ with Lebesgue measure admits a Fock representation which is the renormalized square of white noise algebra over $\mathbb{R}^{d}$.

The square oscillator algebra is canonically isomorphic to (a central extension of) the $*$-Lie algebra $\operatorname{sl}(2, \mathbb{R})$. This is easily seen because $s l(2, \mathbb{R})$ is the $*$-Lie algebra with 3 generators $B^{-}, B^{+}, M$ and relations

$$
\begin{gathered}
\left(B^{-}\right)^{*}=B^{+} ; \quad N^{*}=N \\
{\left[B^{-}, B^{+}\right]=M} \\
{\left[M, B^{ \pm}\right]= \pm 2 B^{ \pm}}
\end{gathered}
$$


From this it follows that the algebra of the RSWN is isomorphic to The Fock representation of a current algebra over a central extension of $\operatorname{sl}(2, \mathbb{R})$.

Example (4) The Schrödinger algebra is the $*$-Lie algebra with generators $\left\{a^{+}, a a^{+2}, a^{2}, a^{+} a, 1\right\}$ and relations deduced from (57).

Example (5) The full oscillator algebra is the $*$-Lie algebra with set of generators $\left\{a^{+h} a^{k}: \forall h, k \in \mathbb{N}\right\}$ and relations deduced from (57).

Notice that all the $*$-Lie algebras listed in the above examples admit a Fock representation. In section (21) we will see that this is not true for the associated current algebra over $\mathbb{R}^{d}$ with Lebesgue measure (for any $d>0$ ).

\section{Connections with classical independent increment processes}

In this section we look for some necessary conditions for the solution of the problem stated in the previous section. This will naturally lead to an interesting connection with the theory of classical independent increment processes which was first noticed in Araki's thesis [Arak60]. We refer to the monographs of K.R. Parthasarathy and K. Schmidt [PaSch72] and of Guichardet [Gui72] for a systematic exposition. In the notations of section (18) we consider:

- a pair $\left\{\mathcal{G},\left(l_{\alpha}^{\varepsilon}\right)\right\}$ of a $*$-Lie algebra and a set of generators which admits a Fock representation.

- a measure space $(S, \mu)$

- a *-sub-algebra $\mathcal{C} \subseteq L_{\mathbb{C}}^{\infty}(S, \mathcal{B}, \mu)$

such that the current algebra

$$
\left\{l_{\alpha}^{\varepsilon}(f): \varepsilon \in\{+,-, 0\}, \quad \alpha \in I \text { or } \alpha \in I_{0}, \quad f \in \mathcal{C}\right\}
$$

admits a Fock representation on some Hilbert space $\mathcal{H}$ with cyclic vector $\Phi$. We identify the elements of this current algebra with their images in this representation and we omit from the notation the symbol $\pi$ of the representation. Moreover we add the following assumptions:

(i) among the generators $\left(l_{\alpha}^{\varepsilon}\right)$ there is exactly one (self-adjoint) central element, denoted $l_{0}^{0}$.

(ii) for any $f \in \mathcal{C}$ one has:

$$
l_{0}^{0}(f)=\int_{S} f d \mu
$$

where the scalar on the right hand side is identified to the corresponding multiple of the identity operator on $\mathcal{H}$. In particular the representation is weakly irreducible.

Under these conditions it is not difficult to see that the general principle that algebra implies statistics can be applied and that the vacuum mixed moments of the operators $l_{\alpha}^{\varepsilon}(f)$ are uniquely determined by the structure 
constants of the Lie algebra. Another important property is that, by fixing a measurable subset $I \subseteq S$ such that

$$
\mu(I)=1
$$

and denoting $\chi_{I}$ the corresponding characteristic function, the $*$-Lie algebra generated by the operators $l_{\alpha}^{\varepsilon}\left(\chi_{I}\right)$ is isomorphic to $\mathcal{G}$ and therefore it has the same vacuum statistics.

Finally the commutation relations (55) imply that the maps $f \mapsto l_{\alpha}^{\varepsilon}(f)$ define an independent increment process of boson type, i.e. the restriction of the vacuum state on the polynomial algebra generated by two families $\left(l_{\alpha}^{\varepsilon}(f)\right)_{\varepsilon, \alpha}$ and $\left(l_{\alpha}^{\varepsilon}(g)\right)_{\varepsilon, \alpha}$ with $f$ and $g$ having disjoint supports, coincides with the tensor product of the restrictions on the single algebras.

In particular, if $X(I)$ is any self-adjoint linear combination of operators of the form $l_{\alpha}^{\varepsilon}\left(\chi_{I}\right)$, then the map $I \subseteq S \mapsto X(I)$ defines an additive independent increment process on $(S, \mathcal{B}, \mu)$. Thus the law of every random variable of the form $X(I)$ will be an infinitely divisible law on $\mathbb{R}$ whenever the set $I$ can be written as a countable union of subsets of nonzero $\mu$-measure.

If $S=\mathbb{R}^{d}$ and $\mu$ is the Lebesgue measure, then any such process $X(I)$ $\left(I \subseteq \mathbb{R}^{d}\right)$ will also be translation invariant.

Combining together all the above remarks one obtains a necessary condition for the existence of the Fock representation of the current algebra of a *-Lie algebra $\mathcal{G}$ and a set of generators namely: the pair $\left\{\mathcal{G},\left(l_{\alpha}^{\varepsilon}\right)\right\}$ must admit a Fock representation and the vacuum distribution of any self-adjoint linear combination $X$ of generators must be infinitely divisible.

Since there is no reason to expect that any pair $\left\{\mathcal{G},\left(l_{\alpha}^{\varepsilon}\right)\right\}$ will have this property, this gives a probabilistic intuition of the reason why it might happen that a $*$-Lie algebra and a set of generators $\left\{\mathcal{G},\left(l_{\alpha}^{\varepsilon}\right)\right\}$ might admit a Fock representation without this being true for the associated current algebra.

In the following section we review some progresses made in the past few years in one important special case: the full oscillator algebra.

\section{Current algebras over the full oscillator algebra}

We have seen how the developments reviewed in the previous sections naturally lead to the following problem: can we extend to the renormalized higher powers of quantum white noise what has been achieved for the second powers? To answer this question we start with the Heisenberg algebra

$$
\left[a, a^{+}\right]=1
$$

Its universally enveloping algebra is generated by the products of monomials of the form

$$
a^{n} \quad, \quad a^{+m}
$$


and their commutation relations are deduced from (63) and the derivation property of the commutator. The problem we want to study is the following: does there exist a current representation of this algebra over $\mathbb{R}^{d}$ for some $d>0$ ?

In order to define the current algebra of the full oscillator algebra, we have first to overcome the renormalization problem, illustrated in section (14) in the case of the second powers of white noise. In fact, dealing with higher powers of white noise we meet higher powers of the $\delta$-function. A natural way out is to write

$$
\delta^{n}=\delta^{2}\left(\delta^{n-2}\right) \quad ; \quad n \geq 2 \quad ; \quad \delta^{0}:=1
$$

and to apply iteratively the renormalization prescription used in section (14). This leads to the following:

Definition The boson Fock white noise, renormalized with the prescription:

$$
\delta(t)^{l}=c^{l-1} \delta(t) \quad, c>0 \quad, l=2,3, \ldots .
$$

simply called RBFWN in the following, over a Hilbert space $\mathcal{H}$ with vacuum (unit) vector $\Phi$ is the locally finite $*$-Lie algebra canonically associated to the associative unital $*$-algebra of operator-valued distributions on $\mathcal{H}$ with generators

$$
b_{t}^{+n} b_{t}^{k} \quad, \quad k, n \in \mathbb{N} \quad, \quad t \in \mathbb{R}^{d}
$$

and relations deduced from:

$$
\begin{gathered}
{\left[b_{t}, b_{s}^{+}\right]=\delta(t-s)} \\
{\left[b_{t}^{+}, b_{s}^{+}\right]=\left[b_{t}, b_{s}\right]=0} \\
\left(b_{s}\right)^{*}=b_{s}^{+} \\
b_{t} \Phi=0
\end{gathered}
$$

Here locally finite means that the commutator of any pair of generators is a finite linear combination of generators.

Lemma The $*$-Lie algebra, associated to the RBFWN (renormalized boson Fock white noise), is the Lie algebra with generators

$$
b_{t}^{+k} b_{t}^{n}=: b_{n}^{k}(t)
$$

central element $b_{t}^{0} b_{t}^{+0}=: 1$ and relations

$$
\begin{gathered}
\left(b_{s}^{+k} b_{t}^{n}\right)^{+}=\left(b_{t}^{+n}\right) b_{s}^{k} \\
{\left[b_{t}^{n}, b_{s}^{+k}\right]=\epsilon_{n, 0} \epsilon_{k, 0} \sum_{l \geq 1}\left(\begin{array}{c}
n \\
l
\end{array}\right) k^{(l)} c^{l-1} b_{s}^{+k-l} b_{t}^{n-l} \delta(t-s)}
\end{gathered}
$$

where: 


$$
\begin{gathered}
k=0,1,2, \ldots \\
\epsilon_{n, k}:=1-\delta_{n, k} \\
k^{(l)}=k(k-1)(k-2) \cdots(k-l+1)
\end{gathered}
$$

and, if $l>k$, by definition

$$
\begin{aligned}
& k^{(l)}=0 \\
& \left(\begin{array}{l}
n \\
l
\end{array}\right)=0
\end{aligned}
$$

In our case we fix the test function space to be the algebra of finite step functions. In terms of test functions we have the identifications:

$$
\begin{gathered}
B_{k}^{n}(f)=\int_{\mathbb{R}^{d}} f(t) b_{t}^{+n} b_{t}^{k} d t \\
\left(B_{k}^{n}(f)\right)^{*}=B_{n}^{k}(\bar{f})
\end{gathered}
$$

\section{No-go theorems}

The first no-go theorem, showing that it is not true that, if a Lie algebra admits a Fock representation, then any associated current algebra also admits one was proved by Sniady [Śnia99]. In the terminology introduced in the present paper Sniady's result can be rephrased as follows:

Theorem 10. The Schrödinger algebra admits a Fock representation but its associated current algebra over $\mathbb{R}$ with Lebesgue measure doesn't.

Since the Schrödinger algebra is contained in the full oscillator algebra, which clearly admits a Fock representation, Sniady's theorem also rules out the possibility of a Fock representation for the current algebra of the full oscillator algebra over $\mathbb{R}$ with Lebesgue measure.

Recalling, from the examples at the end of section (18), that the Schrödinger algebra is the smallest $*$-Lie algebra containing the oscillator algebra (with generators $\left.\left\{a^{+}, a, a^{+} a, 1\right\}\right)$ and the square-oscillator algebra, i.e. $\operatorname{sl}(2, \mathbb{R})$ (with generators $\left\{a^{+2}, a^{2}, a^{+} a, 1\right\}$ ), we see that the difficulty comes from the combination of two closed Lie algebras. More precisely: consider the two sets of generators

$$
\begin{gathered}
\left\{a^{+}, a, a^{+} a, 1\right\} \\
\left\{a^{+2}, a^{2}, a^{+} a, 1\right\}
\end{gathered}
$$

We know that the current algebra over $\mathbb{R}^{d}$ associated to each of them has a Fock representation. However the union of the two sets, i.e.

$$
\left\{a^{+}, a, a^{+2}, a^{2}, a^{+} a, 1\right\}
$$


is also a set of generators of a $*-$ Lie algebra whose associated current algebra over $\mathbb{R}^{d}$ does not admit a Fock representation.

Notice that the first of the two algebras is generated by the first powers of the white noise and the number operator while the second one is generated by the second powers of the white noise and the number operator. An extrapolation of this argument suggested the hope that a similar thing could happen also for the higher powers, i.e. that, denoting $\mathcal{G}_{3}$ the $*$-Lie algebra generated by the cube of the white noise $b_{t}^{3}$ and the number operator; and, for $n \geq 4, \mathcal{G}_{n}$ the $*$-Lie algebra generated by the number operator and the smallest power of the white noise not included in $\bigcup_{1 \leq k \leq n-1} \mathcal{G}_{k}$, the current algebra of $\mathcal{G}_{n}$ over $\mathbb{R}^{d}$ admits a Fock representation.

This hope was ruled out by the following generalization of Sniady's theorem, due to Accardi, Boukas and Franz [AcBouFr05] and by its corollary reported below.

Theorem 11. Let $\mathcal{L}$ be a Lie $*$-algebra with the following properties:

(i) $\mathcal{L}$ contains $B_{0}^{n}$, and $B_{0}^{2 n}$ where by definition:

$$
B_{k}^{n}:=\int_{I} b_{t}^{+n} b_{t}^{k} d t \quad ; \quad I \subseteq \mathbb{R}^{d}
$$

(ii) the $B_{K}^{N}$ satisfy the commutation relations of the higher powers of white noise.

Then, in the notation (65), $\mathcal{L}$ has not a Fock representation if the interval I is such that

$$
\mu(I) \leq \frac{1}{c}
$$

where $c$ is the renormalization constant.

Corollary 12. The current algebra over $\mathbb{R}^{d}$ of the $*-$ Lie algebra $\mathcal{G}_{3}$, generated by the cube of the quantum white noise $b_{t}^{3}$ and the number operator, does not admit a Fock representation if, in the notation (65), the interval I is such that

$$
\mu(I) \leq \frac{1}{c}
$$

where $c$ is the renormalization constant.

Idea of the proof. One proves that, if a Lie algebra contains $b_{t}^{3}$ and $b_{t}^{+3}$, then it contains $b_{t}^{6}$ hence $b_{t}^{+6}$. The thesis then follows from Theorem (11).

Theorem 13. Let $\mathcal{L}$ be a Lie $*$-subalgebra of the RPWN Lie algebra which contains $B_{0}^{n}$ for some $n \geq 3$. Then $\mathcal{L}$ does not admit a Fock space representation if, in the notation (65), the interval I is such that

$$
\mu(I) \leq \frac{1}{c}
$$

where $c$ is the renormalization constant. 
Another hope was that maybe the root of the difficulty is in the insistence on the Fock representation and that maybe the analogue of the finite temperature (equilibrium) representations might exist.

The problem here is that, at the moment there is no general structure theory of free dynamics and of the associated KMS (Kubo-Martin-Schwinger) states on current subalgebras of the full oscillator algebra.

In the case of the square of white noise a classification of free dynamics was obtained and a first class of examples of KMS states was built in the paper [AcAmFr02] and A more general class of KMS functionals was built in the paper [AcPeRo05] but a proof of the positivity of these functionals was missing. The proof of the positivity of these functionals was obtained for an interesting and physically meaningful class of functionals by Prohorenko [Prohor05]

The following theorem, also proved in [AcBouFr05], concerns the non compatibility between first and second order white noise dropping the Fock assumption and replacing it by the use of the natural commutation relations between first and second order noise.

Theorem 14. Let $\mathcal{A}$ be a $*$-algebra of operator valued distributions on $\mathbb{R}^{d}$ whose test function space includes the characteristic functions of intervals in $\mathbb{R}^{d}$ defined as follows:

$$
(a, b)=\left\{\begin{array}{l}
\left\{x=\left(x_{j}\right): a_{j}<x_{j}<b_{j} ; \quad j=1, \ldots, d\right\}, \text { if } a_{j}<b_{j}, \forall j \\
\phi, \text { if } a_{j}>b_{j} \text { for some } j
\end{array}\right.
$$

and let

$$
b_{k}^{2}, b_{k}^{+2}, b_{h}, b_{h}^{+}
$$

be elements of $\mathcal{A}$. There exists no state $\langle\cdot\rangle$ on $\mathcal{A}$ with the following properties:

$$
\begin{gathered}
\left\langle b_{k} b_{h}^{+}\right\rangle=\mu(k) \delta(k-h) \\
\left\langle b_{k^{\prime}} b_{k} b_{h^{\prime}}^{+} b_{h}^{+}\right\rangle=2 \mu(k) \mu\left(k^{\prime}\right) \delta\left(k^{\prime}-h^{\prime}\right) \delta(k-h) \\
\left\langle b_{k}^{2} b_{h^{\prime}}^{+} b_{h}^{+}\right\rangle=2 \mu(k)^{2} \delta\left(k-h^{\prime}\right) \delta(k-h) \\
\left\langle b_{k}^{2} b_{h}^{+2}\right\rangle=\sigma(k) \delta(k-h)
\end{gathered}
$$

where $\sigma \in L_{\text {loc }}^{1}\left(\mathbb{R}^{d}\right)$ and $\mu \in L_{\text {loc }}^{1} \cap L_{\text {loc }}^{2}(\mathbb{R})$ are such that there exist an interval $I \subseteq \mathbb{R}^{d}$ and constants $M_{I}, \varepsilon_{I}>0$ such that

$$
+\infty>M_{I}>\sigma(k), \quad \mu(k) ; \mu(k) \geq \varepsilon_{I}>0 ; \quad \forall k \in I
$$

A third possible loophole is to consider commutation relations not of Boson type. The simplest choice is provided by the $q$-deformed commutation relations: 


$$
a a^{+}-q a^{+} a=1
$$

Bozeiko, Kümmerer and Speicher [BoKüSp96b] proved that this algebra and the associated current algebra over $\mathbb{R}^{d}$, admits a Fock representation if $q \in$ $(-1,1)$ and Bozeiko, and Speicher [BozSpe96a] proved that this algebra and the associated current algebra over $\mathbb{R}^{d}$, does not admits a Fock representation if $|q|>1$.

Theorem 15. ([AcBou05a]) Let $q \in(-1,1), q \neq 0$ and for a fixed interval $I \subset \mathbb{R}$ and $n, k \geq 0$ let $\mathcal{L}_{q}$ denote the $*$-Lie algebra generated by the operators

$$
B_{k}^{n}:=B_{k}^{n}\left(\chi_{I}\right)
$$

defined as in (65) but for the q-deformed white noise. Then $\mathcal{L}_{q}$ does not admit a Fock representation if, the interval $I$ is such that

$$
\mu(I) \leq \frac{1}{c}
$$

where $c$ is the renormalization constant.

Remark. It is interesting to notice that the lower bound $1 / c$ seems to be universal, i.e. independent of the type of noise considered.

\section{Connection with an old open problem in classical probability}

Since the vacuum distribution of the first order classical white noise is a Gaussian, any reasonable renormalization should lead to the conclusion that the $n$-th power of the first order classical white noise is still the $n$-th power of a Gaussian. But the $\delta$-correlation implies that the corresponding integrated process will be a stationary additive independent increment process on $\mathbb{R}$.

These heuristic ideas, which can be put in a satisfactory mathematical form with some additional work, lead to the conjecture that a necessary condition for the existence of the $n$-th power of white noise, renormalized as in [AcBouFr05], is that the $n$-th power of a classical Gaussian random variable is infinitely divisible.

The $n$-th powers of the standard Gaussian random variable $\gamma$ and their distributions have been widely studied. It is known that, $\forall k \geq 1 \gamma^{2 k}$ is infinitely divisible, but it is not known if, $\forall k \geq 1 \gamma^{2 k+1}$ is infinitely divisible (and the experts conjecture that, at least for $\gamma^{3}$, the answer is negative).

\section{Renormalized powers of white noise and the Virasoro-Zamolodchikov algebra}

In the present section we will use the notations of section (20) and the results of the papers [AcBou06a], [AcBou06b], [AcBou06c] which contain the proofs of all the results discussed here. 
The formal extension of the white noise commutation relations to the associative $*$-algebra generated by $b_{t}, b_{s}^{\dagger}, 1$, called from now on the renormalized higher powers of (Boson) white noise (RHPWN) algebra, leads to the identities:

$$
\begin{aligned}
{\left[b_{t}^{\dagger} b_{t}^{k}, b_{s}^{\dagger} b_{s}^{K}\right] } & =\epsilon_{k, 0} \epsilon_{N, 0} \sum_{L \geq 1}\left(\begin{array}{c}
k \\
L
\end{array}\right) N^{(L)} b_{t}^{\dagger^{n}} b_{s}^{\dagger^{N-L}} b_{t}^{k-L} b_{s}^{K} \delta^{L}(t-s) \\
& -\epsilon_{K, 0} \epsilon_{n, 0} \sum_{L \geq 1}\left(\begin{array}{c}
K \\
L
\end{array}\right) n^{(L)} b_{s}^{\dagger^{N}} b_{t}^{\dagger^{n-L}} b_{s}^{K-L} b_{t}^{k} \delta^{L}(t-s)
\end{aligned}
$$

In section (20) we have given a meaning to these formal commutation relations, i.e. to the ill defined powers of the $\delta$-function, through the renormalization prescription (64).

In the present note we will use a different renormalization rule, introduced in [AcBou06a] and whose motivations are discussed in [AcBou06b], [AcBou06c], namely:

$$
\delta^{l}(t-s)=\delta(s) \delta(t-s), \quad l=2,3,4, \ldots
$$

where the right hand side is defined as a convolution of distributions. Using this (78) can be rewritten in the form:

$$
\begin{aligned}
{\left[b_{t}^{\dagger} b_{t}^{k}, b_{s}^{\dagger} b_{s}^{K}\right]=} & \\
& \epsilon_{k, 0} \epsilon_{N, 0}\left(k N b_{t}^{\dagger^{n}} b_{s}^{\dagger^{N-1}} b_{t}^{k-1} b_{s}^{K} \delta(t-s)\right. \\
+ & \left.\sum_{L \geq 2}\left(\begin{array}{c}
k \\
L
\end{array}\right) N^{(L)} b_{t}^{\dagger^{n}} b_{s}^{\dagger^{N-L}} b_{t}^{k-L} b_{s}^{K} \delta(s) \delta(t-s)\right) \\
& -\epsilon_{K, 0} \epsilon_{n, 0}\left(K n b_{s}^{\dagger^{N}} b_{t}^{\dagger^{n-1}} b_{s}^{K-1} b_{t}^{k} \delta(t-s)\right. \\
& \left.+\sum_{L \geq 2}\left(\begin{array}{c}
K \\
L
\end{array}\right) n^{(L)} b_{s}^{\dagger^{N}} b_{t}^{\dagger n-L} b_{s}^{K-L} b_{t}^{k} \delta(s) \delta(t-s)\right)
\end{aligned}
$$

Introducing test functions and the associated smeared fields

$$
B_{k}^{n}(f):=\int_{\mathbb{R}} f(t) b_{t}^{\dagger} b_{t}^{k} d t
$$

the commutation relations (79) become:

$$
\begin{gathered}
{\left[B_{k}^{n}(\bar{g}), B_{K}^{N}(f)\right]=\left(\epsilon_{k, 0} \epsilon_{N, 0} k N-\epsilon_{K, 0} \epsilon_{n, 0} K n\right) B_{K+k-1}^{N+n-1}(\bar{g} f)} \\
\quad+\sum_{L=2}^{(K \wedge n) \vee(k \wedge N)} \theta_{L}(n, k ; N, K) \bar{g}(0) f(0) b_{0}^{\dagger+n-l} b_{0}^{K+k-l}
\end{gathered}
$$




$$
\theta_{L}(N, K ; n, k):=\epsilon_{K, 0} \epsilon_{n, 0}\left(\begin{array}{c}
K \\
L
\end{array}\right) n^{(L)}-\epsilon_{k, 0} \epsilon_{N, 0}\left(\begin{array}{c}
k \\
L
\end{array}\right) N^{(L)}
$$

The commutation relations (81) still contain the ill defined symbols $b_{0}^{ \pm N+n-l}$. However, if the test function space is chosen so that

$$
f(0)=g(0)=0
$$

then the singular term in (81) vanishes and the commutation relations (81) become:

$$
\left[B_{k}^{n}(\bar{g}), B_{K}^{N}(f)\right]_{R}:=(k N-K n) B_{k+K-1}^{n+N-1}(\bar{g} f)
$$

which no longer include ill defined objects. In the following, the symbol $[\cdot, \cdot]_{R}$ denotes these renormalized commutation relations.

A direct calculation shows that the commutation relations (84) define, on the family of symbols $B_{k}^{n}(f)$, a structure of $*$-Lie algebra with involution

$$
B_{k}^{n}(f)^{*}:=B_{n}^{k}(\bar{f})
$$

The commutation relations (84) imply that, fixing a sub-set $I \subseteq \mathbb{R}^{d}$, not containing 0 , and the test function

$$
\chi_{I}(s)= \begin{cases}1, & s \in I \\ 0, & s \notin I\end{cases}
$$

the (self-adjoint) family

$$
\left\{B_{k}^{n}:=B_{k}^{n}\left(\chi_{I}\right): n, k \in \mathbb{N}, n, k \geq 1, n+k \geq 3\right\}
$$

satisfies the commutation relations

$$
\left[B_{k}^{n}, B_{K}^{N}\right]_{R}:=(k N-K n) B_{k+K-1}^{n+N-1}
$$

The comments to condition (62) then suggest the natural interpretation of the *-Lie-algebra, defined by the relations (86), (87), as the 1-mode algebra of the RHPWN and, conversely, the interpretation of the RHPWN *-Lie-algebra as a current algebra of its 1 -mode version.

Now recall the following definition (see, for example, [Ketov95], [Pope91]):

Definition 16. The $w_{\infty}-*-L i e-a l g e b r a$ is the infinite dimensional Lie algebra spanned by the generators $\hat{B}_{k}^{n}$, where $n, N \in \mathbb{N}, n, N \geq 2$ and $k, K \in \mathbb{Z}$, with commutation relations:

$$
\left[\hat{B}_{k}^{n}, \hat{B}_{K}^{N}\right]_{w_{\infty}}=(k(N-1)-K(n-1)) \hat{B}_{k+K}^{n+N-2}
$$

and involution

$$
\left(\hat{B}_{k}^{n}\right)^{*}=\hat{B}_{-k}^{n}
$$


Remark 17. The $w_{\infty}-*$-Lie-algebra, whose elements are interpreted as area preserving diffeomorphisms of 2-manifolds, contains as a sub-Lie-algebra (not as $*$-sub-Lie-algebra) the (centerless) Virasoro (or Witt) algebra with commutations relations

$$
\left[\hat{B}_{k}^{2}(\bar{g}), \hat{B}_{K}^{2}(f)\right]_{V}:=(k-K) \hat{B}_{k+k}^{2}(\bar{g} f)
$$

Both $w_{\infty}$ and a quantum deformation of it, denoted $W_{\infty}$, have been studied extensively in connection to two-dimensional Conformal Field Theory and Quantum Gravity.

The striking similarity between the commutation relations (88) and (87) suggests that the two algebras are deeply related. The following theorem shows that the current algebra, over $\mathbb{R}$, of the $w_{\infty}-*-$ Lie-algebra can be realized in terms of the renormalized powers of white noise.

Theorem 18. Let $\mathcal{S}_{0}$ be the test function space of complex valued (rightcontinuous) step functions on $\mathbb{R}$ assuming a finite number of values and vanishing at zero, and let the powers of the $\delta$-function be renormalized by the prescription

$$
\delta^{l}(t-s)=\delta(s) \delta(t-s), \quad l=2,3, \ldots
$$

Then the white noise operators

$\hat{B}_{k}^{n}(f):=\int_{\mathbb{R}} f(t) e^{\frac{k}{2}\left(b_{t}-b_{t}^{\dagger}\right)}\left(\frac{b_{t}+b_{t}^{\dagger}}{2}\right)^{n-1} e^{\frac{k}{2}\left(b_{t}-b_{t}^{\dagger}\right)} d t ; n \in \mathbb{N}, n \geq 2, k \in \mathbb{Z}$

satisfy the relations (88) and (89) of the $w_{\infty}$-Lie algebra.

Remark 19. The integral on the right hand side of (91) is meant in the sense that one expands the exponential series, applies the commutation relations (78) to bring the resulting expression to normal order, introduces the renormalization prescription (90) and integrates the resulting expressions after multiplication by a test function. The proof shows in particular that the result is an element of the RHPWN $*-$ Lie-algebra.

\section{Bibliography}

[Itô42b] K. Itô: Differential equations determining a Markoff process (in Japanese), Journ. Pan-Japan Math. Coll. 1077 (1942); (in English)

[Itô51] K. Itô: On stochastic differential equations, Mem. Amer. Math. Soc. 4, 1-51 (1951)

[ItôMcKn65] K. Itô and H.P. McKean, Jr.: Diffusion Processes and Their Sample Paths, Springer-Verlag (1965); in Classics in Mathematics, SpringerVerlag (1996) 
[AcLuVo02] Accardi L., Lu Y.G., Volovich I.: Quantum Theory and its Stochastic Limit, Springer Verlag (2002)

[AcBou06a] Accardi L., Boukas A., Renormalized Higher Powers of White Noise (RHPWN) and Conformal Field Theory submitted to: IDA-QP (Infinite Dimensional Analysis, Quantum Probability and Related Topics), Preprint Volterra (2006)

[AcBou06b] Accardi L., Boukas A., The emergence of the Virasoro and $w_{\infty}$ algebras through the renormalized powers of quantum white noise, submitted to: International Journal of Mathematics and Computer Science Preprint Volterra (2006)

[AcBou06c] Accardi L., Boukas A., Lie algebras associated with the RHPWN, submitted to: Communications on Stochastic Analysis (COSA), Preprint Volterra (2006)

[AcBouFr06] Accardi L., Boukas A., Franz U.: Renormalized powers of quantum white noise, IDA-QP (Infinite Dimensional Anal. Quantum Probab. Related Topics) 9 (1) (2006) 129-147 Preprint Volterra n. 597 (2006)

[AcKuSt05a] Accardi L., Kuo H.-H., Stan A.: Moments and commutators of probability measures Preprint Volterra (2005)

[AcKuSt02] Accardi L., Kuo H.-H., Stan A.: Orthogonal polynomials and interacting Fock spaces, IDA-QP (Infinite Dimensional Anal. Quantum Probab. Related Topics) 7 (4) (2004) 485-505

[AcBou05a] Accardi L., Boukas A., Higher Powers of $q$-deformed White Noise, submitted to: Methods of Functional Analysis and Topology (2005)

[AcBou04c] L. Accardi, Andreas Boukas: White noise calculus and stocastic calculus Talk given at: International Conference on "Stochastic analysis: classical and quantum, Perspectives of white noise theory", Meijo University, Nagoya, November 1-5, 2003 in: Quantum Information, T. Hida, K. SaItô (eds.) World Scientific (2005) 260-300 Preprint Volterra n. 579 (2005)

[AcBou03] Accardi L., Boukas A.: Unitarity conditions for the renormalized square of white noise, Infinite Dimensional Anal. Quantum Probab. Related Topics 6 (2) (2003) 197-222

[AcRosc05] L. Accardi, R. Roschin: Renormalized squares of Boson fields, IDA-QP (Infinite Dimensional Analysis, Quantum Probability and Related Topics) 8 (2) (2005) 307-326

[AcAmFr02] Luigi Accardi, Grigori Amosov, Uwe Franz: Second quantized automorphisms of the renormalized square of white noise (RSWN) algebra IDA-QP (Infinite Dimensional Analysis, Quantum Probability and Related Topics) 7 (2) (2004) 183-194 Preprint Volterra (2002)

[AcBou01a] Accardi L., Boukas A.: Square of white noise unitary evolutions on Boson Fock space, in: Proceedings International conference on stochastic analysis in honor of Paul Kree, Hammamet, Tunisie, October 22-27, 2001, S. Albeverio, A. Boutet de Monvel, H. Oueridiane (eds.) Kluwer (2004) 267-302

[AcLuVo99] Accardi L., Lu Y.G., Volovich I.V.: White noise approach to classical and quantum stochastic calculi, Lecture Notes of the Volterra-CIRM 
International School with the same title, Trento, Italy, 1999, Volterra Preprint N. 375 July (1999)

[AcAyOu05a] Accardi L., Ayed W., Ouerdiane H.: White noise Heisenberg evolution and Evans-Hudson flows, to appear in: IDA-QP (Infinite Dimensional Analysis, Quantum Probability and Related Topics), Preprint Volterra n. $590(2005)$

[AcAyOu05b] Accardi L., Ayed W.: Module Form of The White Noise Stochastic Calculus, submitted to: IDA-QP (Infinite Dimensional Analysis, Quantum Probability and Related Topics), Preprint Volterra (2005)

[AcAyOu03] Luigi Accardi, Wided Ayed, Habib Ouerdiane: White Noise Approach to Stochastic Integration, talk given (by W.A.) at the Colloque International de Mathematiques, Analyse et Probabilites, 20-25 Octobre 2003, Hammamet, (Tunisie); Random Operators and Stochastic Equations 13 (4) (2005) 369-398 Preprint Volterra n. 573 (2004)

[AcBo98] Accardi L., Bożejko M.: Interacting Fock spaces and Gaussianization of probability measures, Infinite dimensional analysis, quantum probability and related topics, 1, N. 4 (1998) 663-670

[AcLuVo97b] Accardi L., Lu Y.G., Volovich I.: The QED Hilbert module and Interacting Fock spaces, Publications of IIAS (Kyoto) (1997)

[AcLuVo93] Accardi L., Lu Y.G., Volovich I.: The Stochastic Sector of Quantum Field Theory, Matematicheskie Zametki (1994) Volterra Preprint N. 138 (1993)

[AcHud89] Accardi L., Hudson R.: Quantum stochastic flows and non abelian cohomology, in: Quantum Probability and Applications V, Springer LNM 1442 (1990) Volterra preprint N. 16 (1989)

[AcFrLu87] Accardi L., Frigerio A., Lu Y.G.: On the weak coupling limit problem, in: [QP-PQ IV], Quantum Probability and Applications IV Springer LNM N. 1396 (1987) 20-58

[Ac78b] Accardi L.: On the quantum Feynmann-Kac formula, Rendiconti del seminario Matematico e Fisico, Milano 48 (1978) 135-179

[Arak60] Araki H.: Princeton Thesis (1960)

[Ayed05] Ayed Wided: White Noise Approach to Quantum Stochastic Calculus, Thesis, University Tunis El Manar dan University, Tor Vergata (2005)

[Barc88] Barchielli A.: Input and output channels in quantum systems and quantum stochastic differential equations, in: [QP-PQ III], SLNM 1303 (ed) Accardi L. von Waldenfels W, Springer (1988)

[Bela86a] Belavkin V.P.: Optimal linear filtering and control in quantum linear systems, In: Proc of I F A C Conference on Statistical Control Theory, Vilnius (1986)

[BogLogTod69] Bogolyubov, N. N., Logunov, A. A., Todorov, I. T.: Fundamentals of the axiomatic approach in quantum field theory (Russian) [Izdat. "Nauka", Moscow (1969) English translation: Bogolubov, N. N.; Logunov, A. A.; Todorov, I. T.: Introduction to axiomatic quantum field theory, Mathematical Physics Monograph Series, No. 18. W. A. Benjamin Inc.(1975) 
[BoKüSp96b] Bozejko M., Kümmerer B., Speicher R.: q-Gaussian processes: non commutative and classical aspects, Comm. Math. Phys. 185 (1997) 129-154 Preprint (1996)

[BozSpe96a] Bozejko M., Speicher R.: Interpolations between bosonic and Fermionic relations given by generalized Brownian motions, Math. Zeit. 322 (1996) 135-160

[FrGo82a] Frigerio A., Gorini V.: On stationary Markov dilations of quantum dynamical semigroups, In: [QP-PQ II], Quantum Probability and Applications to the Quantum Theory of Irreversible Processes (a cura di L. Accardi, A. Frigerio, V. Gorini), Proceedings, Villa Mondragone (1982), Springer LNM 1055 (1984) 119-125

[GaCo85] Gardiner C.W., Collet M.J.: Input and output in damped quantum systems: quantum stochastic differential equation and master equation, Phys. Rev. A 31 (1985) 3761-3774

[Grig01] B. Grigelionis: Generalized $z$-distributions and related stochastic processes, Lithuanian Math. J. 41 (2001) 303-319

[Grig99] B. Grigelionis: Processes of Meixner type, Lithuanian Math. J. 39 (1999) 33-41.

[Grig00c] B. Grigelionis: On generalized $z$-diffusions, Preprint Vilnius (2000).

[Gui72] Guichardet A.: Symmetric Hilbert spaces and related topics, Lect. Notes Math.: 261, Springer, Berlin (1972)

[Hida92] Hida T.: Selected papers, World Scientific (2001)

[Hida75] Hida T.: Analysis of Brownian Functionals, Carleton Mathematical Lecture notes 13 (1975); 2nd ed., 1978

[HuPa84c] Hudson R.L., Parthasarathy K.R.: Quantum Itô's formula and stochastic evolutions, Commun. Math. Phys. 93 (1984) 301-323

[HuPa82a] Hudson R.L., Parthasarathy K.R.: Construction of quantum diffusions, in: Quantum probability and applications to the quantum theory of irreversible processes, Proc. 2-d Conference: Quantum Probability and applications to the quantum theory of irreversible processes, 6-11, 9 (1982) Villa Mondragone (Rome), Springer LNM N. 1055 (1984)

[Ketov95] Ketov S. V., Conformal field theory, World Scientific (1995)

[KunWat67] H. Kunita and S. Watanabe: On square integrable martingales, Nagoya Math. J. 30, 209-245 (1967)

[Ivanov79] Ivanov, V.K.: The algebra of elementary generalized functions. (Russian) Dokl. Akad. Nauk SSSR 246 (1979), no.4, 805-808. English translation: Soviet Math. Dokl. 20 (1979), no. 3, 553-556.

[Partha92] Parthasarathy K.R.: An introduction to quantum stochastic calculus, Birkhäuser (1992)

[PaSch72] Parthasarathy K.R., Schmidt K.: Positive definite kernels continuous tensor products and central limit theorems of probability theory, Springer Lecture Notes in Mathematics no. 272 (1972)

[Pope91] Pope C. N., Lectures on W algebras and W gravity, Lectures given at the Trieste Summer School in High-Energy Physics, August 1991 
[Prohor05] D.B. Prohorenko: On the positivity of KMS functionals on the algebra of the square od white noise. Preprint 25-4-2005

[Skor84] Skorodod A.V.: Random linear operators, Reidel (1984)

[Śnia99] P. Śniady: Quadratic bosonic and free white noises, Commun. Math. Phys. 211 (3) (2000) 615-628 Preprint (1999)

[Vlad66] Vladimirov, V. S. : (Russian) [Methods in the theory of functions of several complex variables, Izdat. "Nauka", Moscow (1964), English translation: Vladimirov, V. S.: Methods of the theory of functions of many complex variables, Scripta Technica, Inc. Translation edited by Leon Ehrenpreis The M.I.T. Press (1966)

[voWaGi78] von Waldenfels W., Giri N.: An Algebraic Version of the Central Limit Theorem, Z. Wahrscheinlichkeitstheorie verw. Gebiete 42 (1978) $129-134$

[voWa78] von Waldenfels W.: An Algebraic Central Limit Theorem in the Anticommuting Case. Z. Wahrscheinlichkeitstheorie verw. Gebiete 42 (1978) $135-140$ 